\title{
Orbifold string topology
}

\author{
ERNESTO LUPERCIO \\ BERNARDO URIBE \\ Miguel A XicotencATL
}

\begin{abstract}
In this paper we study the string topology (à la Chas-Sullivan) of an orbifold. We define the string homology ring product at the level of the free loop space of the classifying space of an orbifold. We study its properties and do some explicit calculations.
\end{abstract}

55P35; 18D50, 55R35

\section{Introduction}

String topology is the study of the topological properties of the free loop space $\mathcal{L} M$ of a smooth manifold $M$ by the use of methods originating in quantum field string theories and in classical algebraic topology. Here $\mathcal{L} M$ is by definition the $\operatorname{space} \operatorname{Map}\left(S^{1} ; M\right)$ of piecewise smooth maps from the unit circle $S^{1}$ to $M$. This study was initiated by Chas and Sullivan in their seminal paper [7]. In this paper they defined a remarkable product $\circ$ on the homology $H_{*}(\mathcal{L} M)$ of the loop space of a smooth manifold.

The Chas-Sullivan string product on $H_{*}(\mathcal{L} M)$ was only part of a very interesting structure unveiled in their work; for example they defined a degree one map

given by

$$
\Delta: H_{*}(\mathcal{L} M) \rightarrow H_{*+1}(\mathcal{L} M)
$$

$$
\Delta(\sigma)=\rho_{*}(d \theta \otimes \sigma)
$$

where $\rho: S^{1} \times \mathcal{L} M \rightarrow \mathcal{L} M$ is the evaluation map and $d \theta$ is the fundamental class of $S^{1}$. One of the main theorems of [7] is the following:

Theorem 1.1 (Chas-Sullivan [7]) The triple

$$
\left(H_{*}(\mathcal{L} M), \circ, \Delta\right)
$$

is a Batalin-Vilkovisky algebra, namely:

- $\left(H_{*-d}(\mathcal{L} M), \circ\right)$ is a graded commutative algebra.

- $\Delta^{2}=0$. 
- The bracket

$$
\{\alpha, \beta\}=(-1)^{|\alpha|} \Delta(\alpha \circ \beta)-(-1)^{|\alpha|} \Delta(\alpha) \circ \beta-\alpha \circ \Delta(\beta)
$$

makes $H_{*-d}(M)$ into a graded Gerstenhaber algebra (namely it is a Lie bracket which is a derivation on each variable).

This establishes a striking relation between algebraic topology and recent findings in quantum field theory and string theory; see Batalin and Vilkovisky [5] and Getzler [14].

Cohen and Jones [9] discovered that a very rich part of this structure was available at a more homotopy-theoretic level and reinterpreted the BV-algebra structure in terms of an action of the cactus operad on a certain prospectrum associated to $M$. They showed moreover that the Chas-Sullivan string product was the natural product in the Hochschild cohomology interpretation of the homology of the loop space of $M$; see Jones [15]. Cohen and Godin [8] studied interactions with the study of the homology of moduli spaces of Riemann surfaces, establishing a direct connection to topological quantum field theories. Cohen and Godin used the concept of Sullivan chord diagram in their work. Cohen, Jones and Yan [10] provided more explicit calculations of the product by the careful use of the spectral sequence associated to the fibration

$$
\Omega M \longrightarrow \mathcal{L} M \longrightarrow M
$$

induced by the evaluation map. In particular they computed the Chas-Sullivan product on the homology of the free loop space of spheres and complex projective spaces.

In this paper we generalize several of the fundamental results of string topology by showing that they remain true if we replace the manifold $M$ by an orientable orbifold $\mathrm{X}=[M / G]$, where $G$ is a finite group acting by orientation preserving diffeomorphisms on $M$. More precisely the following theorem is the main result in this paper and can be seen as a generalization of Theorem 1.1 to the orbifold context.

Theorem 1.2 Let $\mathrm{X}=[M / G]$ be an orientable orbifold, then

$$
A_{\mathrm{LX}}:=H_{*}\left(\mathcal{L}\left(M \times_{G} E G\right) ; \mathbb{Q}\right)
$$

has the structure of a Batalin-Vilkovisky algebra.

This BV-algebra can be identified in two extreme cases:

- When $G=\{1\}$ and for arbitrary $M$ then $A_{\mathrm{Lx}}$ coincides with the Chas-Sullivan BV-algebra.

- When $M=\left\{m_{0}\right\}$ is a single point and for arbitrary finite $G$ then $A_{\mathrm{Lx}}$ is isomorphic to the center of the group algebra of $G$. 
This paper is organized as follows. In Section 2 we define a topological groupoid that we call the loop groupoid LX (see the paper [16] of the first two authors) of an orbifold groupoid $X$ and prove that is satisfies the basic property

$$
B \mathrm{LX} \simeq \mathcal{L} B \mathrm{X}
$$

where $B$ is the geometric realization functor from groupoids to topological spaces; see Segal [20]. In Section 3 we define the product in $H_{*}\left(\mathcal{L}\left(M \times_{G} E G\right) ; \mathbb{Q}\right)$ that eventually will be part of the BV-algebra structure. In Section 4 we show that there is a version of the ring of Section 3 twisted by discrete torsion which is analogous to the twistings in equivariant $K$-theory of Adem and Ruan [4]. In Section 5 we prove Theorem 1.2 which is our main result. For this purpose we introduce and operad of Sullivan chord diagrams which we call the chord diagram operad and show that it is equivalent to the cactus operad. Finally in Section 6 we perform some explicit computations, for example we show how to use the methods of this paper to compute the Chas-Sullivan product on Lens spaces.

We would like to thank Alejandro Adem, Carl-Friedrich Bödigheimer, Ralph Cohen, Dan Freed, Sam Gitler, Maxim Kontsevich, Jacob Mostovoy, Mainak Poddar, Antonio Ramirez, Yongbin Ruan, Graeme Segal, Jim Stasheff and Dennis Sullivan for relevant conversations regarding this work.

We acknowledge the support of CONACYT (the first and third authors) and of the Universidad de los Andes (the second author).

\section{The free loop space of the classifying space of an orbifold}

\subsection{Orbifolds}

For an introduction to the theory of orbifolds, we refer the reader to Moerdijk [19] or Adem, Leida and Ruan [3]. Here we just recall the basic definitions we will use in this paper.

Following Moerdijk [19], we will use groupoids to study orbifolds. A groupoid is a (small) category in which each arrow is an isomorphism. Whenever we have a groupoid $G$ we will denote by $G_{0}$ the set of all its objects and by $G_{1}$ the set of all its arrows. We will denote by $G_{1} \times_{s} G_{1}$ the subset of $G_{1} \times G_{1}$ consisting pairs of arrows so that the target of the first equals the source of the second. We will write $1_{x}$ to denote the identity arrow for the object $x$ in $\mathrm{G}_{0}$.

We will denote the structure maps by

$$
\mathrm{G}_{1 \mathrm{t}} \times_{\mathrm{s}} \mathrm{G}_{1} \stackrel{m}{\longrightarrow} \mathrm{G}_{1} \stackrel{i}{\longrightarrow} \mathrm{G}_{1} \stackrel{s}{\underset{t}{\longrightarrow}} \mathrm{G}_{0} \stackrel{e}{\longrightarrow} \mathrm{G}_{1}
$$


where $s$ and $t$ are the source and the target maps, $m$ is the multiplication map given by

$$
m(\alpha, \beta)=\beta \circ \alpha,
$$

$e$ is the unit map defined by

$$
e(x)=1_{x},
$$

and $i$ is the inverse map

$$
i(\alpha)=\alpha^{-1}
$$

Definition 2.1 A Lie groupoid is a groupoid $G$ for which, in addition, $G_{0}$ and $G_{1}$ are Hausdorff smooth manifolds, and the structure maps $s, t, m, e$ and $i$ are all smooth. We will also require $s$ and $t$ to be submersions.

We define several kinds of Lie groupoids.

- A Lie groupoid $\mathrm{G}$ is said to be a proper groupoid if the map $(s, t): \mathrm{G}_{1} \longrightarrow \mathrm{G}_{0} \times \mathrm{G}_{0}$ is proper.

- A Lie groupoid $\mathrm{G}$ is said to be a foliation groupoid if for every object $x \in \mathrm{G}_{0}$ its stabilizer $\mathrm{G}_{x}:=\left\{\alpha \in \mathrm{G}_{1} \mid s(\alpha)=t(\alpha)=x\right\}$ is finite.

- A proper foliation Lie groupoid $\mathrm{G}$ will be called an orbifold groupoid.

Example 2.2 Let $G$ be a finite group acting smoothly from the right on a smooth manifold $M$. Then the translation groupoid $M \rtimes G$ is defined by $(M \rtimes G)_{0}=M$, $(M \rtimes G)_{1}=M \times G, s(x, g)=x, t(x, g)=x g, m((x, g),(x g, h))=(x, g h)$, $i(x, g)=\left(x, g^{-1}\right)$ and $e(x)=\left(x, 1_{G}\right)$.

Moerdijk shows [19] that the orbifold groupoids together with smooth functions form a category, and calls a morphism in this category a Morita equivalence if it is fully faithful and essentially surjective in a an appropriate differential-geometric sense. $\mathrm{He}$ then defines the category of orbifolds as the localization of the category of orbifold groupoids at the class of Morita equivalences. If $M$ and $G$ are as in Example 2.2, we will write $[M / G]$ for the translation groupoid $M \rtimes G$ viewed as an object of the orbifold category. Orbifolds of this form are called 'global quotient orbifolds'. For simplicity, we will restrict our attention to such global quotients orbifolds throughout the paper. A reader not familiar with orbifolds may just think of the data of such global quotient orbifold as equivariant data, keeping in mind that there is a notion of equivalence which might identify the data of an action of a group $G$ on $M$ with those of an action of a different group $\Gamma$ on a different manifold $N$.

Segal in [20] defines a functor from the category of all small categories to the category of topological spaces

$$
B: \text { Cat } \longrightarrow \text { Top }
$$


that he calls the classifying space functor. This functor sends transformations of functors to homotopies of continuous mappings.

The classifying space of the orbifold $\mathrm{X}=[M / G]$ is defined as the classifying space $B(M \rtimes G)$ of the category $M \rtimes G$. It is known [19] that $B(M \rtimes G)$ depends only on the orbifold and not on the particular group action used to represent it. For this reason we will denote it also by $B X$.

Let $\Delta^{n}$ be the standard $n$-simplex. If we denote by $\mathrm{X}_{n}$ the space of ordered $n$-tuples of arrows in $M \rtimes G$ such that the target of each arrow is equal to the source of the following one, then it is not hard to see that

$$
\mathrm{X}_{n} \cong M \times G \times \cdots \times G
$$

where $G$ appears exactly $n$ times. The classifying space is defined as a quotient

$$
B \mathrm{X}:=\bigsqcup_{n}\left(\mathrm{X}_{n} \times \Delta^{n}\right) / \sim
$$

where $\sim$ is an equivalence relation defined by Segal [20].

The space $B \mathrm{X}$ is homotopy equivalent to the Borel construction of the $G$-manifold $M$, namely

$$
B \times:=B(M \rtimes G) \simeq M_{G}:=M \times_{G} E G,
$$

where $E G$ is a contractible space where $G$ acts freely and $M \times{ }_{G} E G:=(M \times E G) / G$ is the quotient by the diagonal action. For a proof of this fact we refer the reader to Segal [20]. We will write $[m, \xi]$ to denote the equivalence class of $(m, \xi) \in M \times E G$ under this diagonal action. We will also write $[Y, T]$ to denote the projection of the set $Y \times T \subseteq M \times E G$ to $B \times$

Let $Y$ and $Z$ be two $G$-spaces, and let

$$
e: Z \rightarrow Y
$$

be an equivariant map between them (ie $e(z g)=e(z) g$ for all $z$ and $g$ ). The assignment that associates to a $G$-space its translation groupoid is functorial. In other words $e$ induces a morphism of groupoids which we call again $e$. We will denote by

$$
|e|: Z \times_{G} E G \longrightarrow Y \times_{G} E G
$$

the corresponding map of classifying spaces. 


\subsection{The loop space as a classifying space for the loop orbifold}

Denote by $\mathcal{L} B \mathrm{X}=\mathcal{L}\left(M_{G}\right)$ the free loop space of $B \mathrm{X}$, then $\mathcal{L} B \mathrm{X}$ depends only on $X$. In this section we will find a topological groupoid $L X$ with the property that $B \mathrm{LX} \simeq \mathcal{L} B \mathrm{X}$.

We find the following notation useful. We set

$$
\mathcal{P}_{y}^{x}(M):=\{\gamma:[0,1] \rightarrow M \mid \gamma(0)=x \text { and } \gamma(1)=y\}
$$

that is to say the space of all piecewise smooth paths going from $x \in M$ to $y \in M$.

We define the loop groupoid LX of a global orbifold $X$ (for a definition in the general case see [16] cf [6]). For this we consider the space

$$
\mathcal{P}_{G}(M):=\bigsqcup_{g \in G} \mathcal{P}_{g}(M) \times\{g\}
$$

where $\quad \mathcal{P}_{g}(M):=\{\gamma:[0,1] \rightarrow M \mid \gamma(0) g=\gamma(1)\}=\bigsqcup_{x \in M} \mathcal{P}_{x g}^{x}(M)$

together with the $G$ action given by

$$
\begin{aligned}
\mathcal{P}_{G}(M) \times G & \rightarrow \mathcal{P}_{G}(M) \\
((\gamma, g), h) & \mapsto\left(\gamma_{h}, h^{-1} g h\right)
\end{aligned}
$$

where $\gamma_{h}(m):=\gamma(m) h$. (See Figure 1.) Then we define the loop groupoid as

$$
\mathrm{LX}:=\mathcal{P}_{G}(M) \rtimes G .
$$

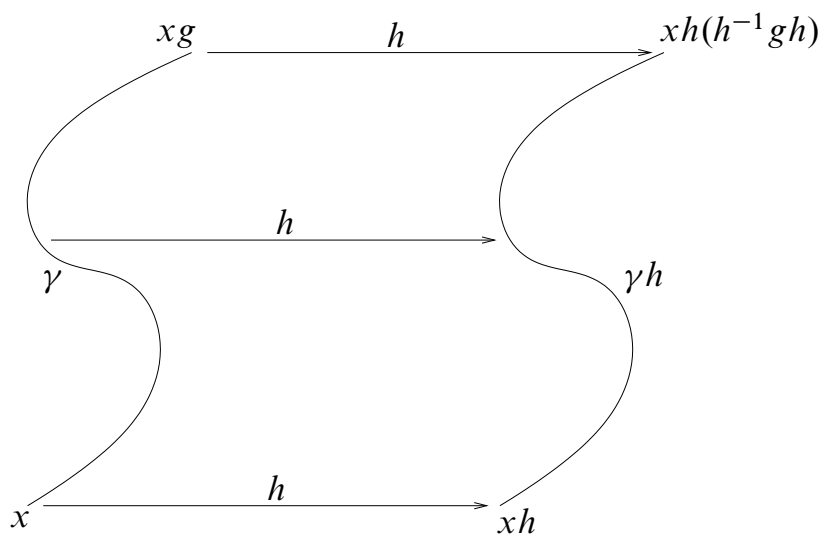

Figure 1 
The following result describes the relation between $\mathcal{L} B X$ and $L X$.

Theorem 2.3 There is a canonical map

$$
\tau: \mathcal{L} B \mathrm{X} \longrightarrow B \mathrm{LX}
$$

that induces a weak homotopy equivalence.

Proof We will construct two Serre fibrations over $B X$.

- Consider $B \mathrm{LX}$. Define a morphism of groupoids

$$
\widetilde{\mathrm{ev}}_{0}: L X \rightarrow X
$$

induced by the equivariant map of $G$-spaces

$$
\mathrm{ev}_{0}: \mathcal{P}_{G}(M) \longrightarrow M
$$

given by evaluation at 0 ,

$$
\mathrm{ev}_{0}(\gamma, g):=\gamma(0)
$$

This morphism induces a map at the level of classifying spaces

$$
\left|\mathrm{ev}_{0}\right|: B \mathrm{LX} \rightarrow B \mathrm{X}
$$

If we interpret the classifying spaces in terms of the Borel construction as in formula (2) we have $B L X=\mathcal{P}_{G}(M) \times_{G} E G$ and $B X=M \times_{G} E G$. For a point $z \in B X$ with $z=[m, \xi]$, the following holds

where

$$
|\mathrm{ev}|^{-1}(z)=\left[\mathcal{P}_{G}^{m}(M) \times\{\xi\}\right]
$$

with

$$
\mathcal{P}_{G}^{m}(M):=\bigsqcup_{g \in G} \mathcal{P}_{g}^{m}(M) \times\{g\}
$$

$$
\mathcal{P}_{g}^{m}(M)=\left\{\gamma \in \mathcal{P}_{g}(M) \mid \gamma(0)=m\right\} .
$$

- On $\mathcal{L} B X$. Take the map

$$
\epsilon_{0}: \mathcal{L} B X \longrightarrow B X
$$

which evaluates a free loop at 0 , ie for $\sigma: S^{1} \rightarrow B \times$ then $\epsilon_{0}(\sigma):=\sigma(0)$. Then

$$
\epsilon_{0}^{-1}(z)=\Omega_{z}(B X):=\mathcal{P}_{z}^{z}(B X)
$$

is the space of loops based at $z$. 
Now let's define the map $\tau$. Consider the fixed $z=[m, \xi]$ as above and for $\sigma \in \mathcal{L} B \mathrm{X}$, lift it to $\widetilde{\sigma}$ making the following diagram commutative

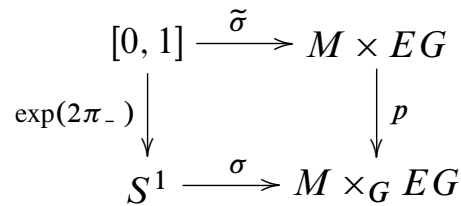

such that $\tilde{\sigma}(0)=(m, \xi)$ (the construction follows from the fact that the map $p$ is a $G$-principal bundle and $G$ is finite). Since $G$ acts freely on $E G$ there exists a unique element $k$ in $G$ such that $\tilde{\sigma}(0) k=\widetilde{\sigma}(1)$. Define $\tau$ in the following way

$$
\tau(\sigma):=\left[\left(\pi_{1} \circ \tilde{\sigma}, k\right), \xi\right] \in B \mathrm{LX},
$$

where $\pi_{1}: M \times E G \rightarrow M$ is the projection on the first coordinate. From the definition of $\tau$ it follows that it is well defined and that $\pi_{1} \circ \tilde{\sigma} \in \mathcal{P}_{k}(M)$. Moreover the following diagram is commutative:

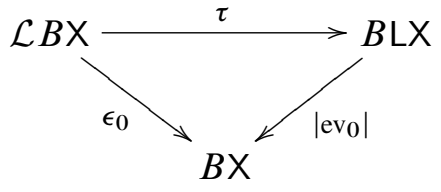

Let's denote by $\tau_{z}:=\left.\tau\right|_{\epsilon_{o}^{-1}(z)}$. Then we have the following lemma:

Lemma 2.4 The map

$$
\tau_{z}: \epsilon_{0}^{-1}(z) \longrightarrow\left|\mathrm{ev}_{0}\right|^{-1}(z)
$$

is a homotopy equivalence.

Proof From the definition of $\tau$ it is clear that $\tau_{z}$ is surjective. Let's now check the homotopy type of the inverse image of a point. Recall from above that the map $\tau_{z}$ goes from $\Omega_{z}\left(M \times_{G} E G\right)$ to $\left[\mathcal{P}_{G}^{m}(M) \times\{\xi\}\right]$. Take $(\gamma, g) \in \mathcal{P}_{G}^{m}(M)$. From the definition of $\tau$ above it follows that

$$
\tau_{z}^{-1}([((\gamma, g), \xi)]) \cong \mathcal{P}_{g^{-1} \xi}^{\xi}(E G)
$$

where $\mathcal{P}_{g^{-1} \xi}^{\xi}(E G)$ stands for the paths in $E G$ that go from $\xi$ to $\left(g^{-1} \xi\right)$.

The space $\mathcal{P}_{g^{-1} \xi}^{\xi}(E G)$ is independent of the choice of representative in $[((\gamma, g), \xi)]$.

As the space $\mathcal{P}_{g^{-1} \xi}^{\xi}(E G)$ is contractible then it follows that $\tau_{z}$ induces a homotopy equivalence. 
As $\tau$ induces a homotopy equivalence on the fibers of the Serre fibrations given by $\epsilon_{0}$ and $\left|\mathrm{ev}_{0}\right|$, then the Theorem 2.3 follows from a theorem of Dold [13]. Hence $\tau$ induces a weak homotopy equivalence between $\mathcal{L} B \mathrm{X}$ and $B \mathrm{LX}$.

\subsection{Circle action}

We have seen that the map $\tau: \mathcal{L} B \mathrm{X} \longrightarrow B(\mathrm{LX})$ is a weak homotopy equivalence, and it is natural to wonder whether the equivalence is $S^{1}$-equivariant. The answer turns out to be negative as we will see shortly.

There is a natural action of $S^{1}$ onto $\mathcal{L} B \mathrm{X}$ by rotating the loop, but the action does not get carried into $B L X$ via $\tau$. The reason is the following, the loop orbifold $L X$ comes provided with a natural action of the orbifold $[\mathbb{R} / \mathbb{Z}]$ which is a stack model for the circle. The action of $\mathbb{R}$ into the orbifold loops of $\mathcal{P}_{G}(M)$ is the obvious one, the map gets shifted by the parameter in $\mathbb{R}$. The subtlety arises here, once we act on the orbifold loop by $1 \in \mathbb{R}$, we do not end up with the orbifold loop from the beginning, but instead we get one that is related to the initial one via an arrow of the loop orbifold category. This arrow in the loop orbifold is where $1 \in \mathbb{Z}$ gets mapped. By the way, precisely this fact was the one that allowed us to define the loop orbifold in a nontrivial way, namely a loop on the orbifold was not a map from the circle to the orbifold, but a functor from $[\mathbb{R} / \mathbb{Z}]$ to the orbifold.

More accurately, to define the action of $[\mathbb{R} / \mathbb{Z}]$ on $L X=\left[\mathcal{P}_{G}(M) / G\right]$ we first define an action of $\mathbb{R}$ on $\mathcal{P}_{G}(M)$ in the natural way, namely, take $\gamma \in \mathcal{P}_{k}(M)$ and $s \in \mathbb{R}$ and define

$$
(s \cdot \gamma)(t):=\gamma_{s}(t)=\gamma(t+s-\lfloor t+s\rfloor) k^{\lfloor t+s\rfloor}
$$

where $\lfloor\cdot\rfloor$ is the least integer function. Then for each $(\gamma, k) \in \mathcal{P}_{G}(M)$ and $1 \in \mathbb{Z}$ we choose the arrow of LX that relates the orbifold loops $(\gamma, k)$ and $\left(\gamma_{1}, k\right)$, this is the arrow $((\gamma, k), k) \in \mathcal{P}_{G}(M) \times G$. The source of $((\gamma, k), k)$ is $(\gamma, k)$ and the target is $(\gamma \cdot k, k)=\left(\gamma_{1}, k\right)$ the loop shifted by 1 .

Using the construction of Section 2.2 we have that

$$
\tau(\sigma):=\left[\left(\left(\pi_{1} \circ \tilde{\sigma}, k\right), \xi\right)\right],
$$

and denote $\pi_{1} \circ \tilde{\sigma}$ by $\gamma$. For $s \in \mathbb{R}$,

$$
\left.\tau(s \cdot \sigma)=\left[\left(\gamma_{s}, k\right), \xi\right)\right]
$$

and $1 \cdot \sigma=\sigma$, but $\tau(1 \cdot \sigma) \neq \tau(\sigma)$. Instead $\tau(1 \cdot \sigma)$ and $\tau(\sigma)$ are related by an arrow. Nevertheless, if we take the coarse moduli space of $\mathrm{LX}$ (that we will write $\mathrm{LX} / \sim=$ $\mathcal{P}_{G}(M) / G$ ), the map induced by $\tau$ is $S^{1}$-equivariant. For in $\mathrm{LX} / \sim=\mathcal{P}_{G}(M) / G$ the elements $\tau(1 \cdot \sigma)$ and $\tau(\sigma)$ become by definition the same. Then we can conclude: 
Lemma 2.5 The space $\mathrm{LX} / \sim=\mathcal{P}_{G}(M) / G$ has a natural $S^{1}$ action and the map

$$
\tilde{\tau}: \mathcal{L} B X \longrightarrow \mathrm{LX} / \sim=\mathcal{P}_{G}(M) / G
$$

which is the composition of $\tau$ with the projection $B \mathrm{LX} \rightarrow \mathrm{LX} / \sim$, is $S^{1}$-equivariant.

Corollary 2.6 The map $\tilde{\tau}$ induces an isomorphism in homology

$$
\tilde{\tau}_{*}: H_{*}(\mathcal{L} B X ; \mathbb{Q}) \stackrel{\cong}{\longrightarrow} H_{*}(\mathrm{LX} / \sim ; \mathbb{Q})
$$

and in equivariant homology

$$
\tilde{\tau}_{*}: H_{*}^{S^{1}}(\mathcal{L} B X ; \mathbb{Q}) \stackrel{\cong}{\longrightarrow} H_{*}^{S^{1}}(\mathrm{LX} / \sim ; \mathbb{Q}) .
$$

Proof As $\tau$ is a weak homotopy equivalence, then

$$
\tau_{*}: H_{*}(\mathcal{L} B \mathrm{X} ; \mathbb{Z}) \stackrel{\cong}{\longrightarrow} H_{*}(B\llcorner\mathrm{X} ; \mathbb{Z}),
$$

and as the group $G$ is finite then

$$
\tilde{\tau}_{*}: H_{*}(B \mathrm{LX} ; \mathbb{Q}) \stackrel{\cong}{\longrightarrow} H_{*}(\mathrm{LX} / \sim ; \mathbb{Q}) .
$$

The second isomorphism follows from the isomorphism of spectral sequences with real coefficients associated to the each of the following fibrations:

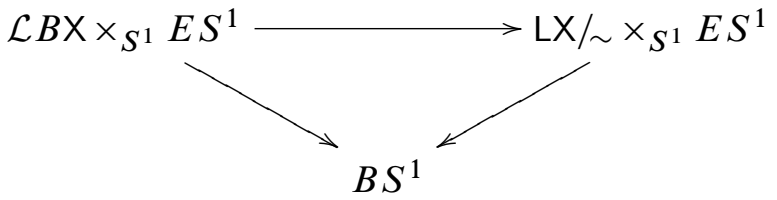

\subsection{Cyclic equivariant loops}

There is an alternative description of $\mathcal{P}_{g}(M)$ that although essentially obvious nevertheless relates it to some models that have been studied before.

Given an element $g \in G$ it generates a cyclic group $\langle g\rangle \subseteq G$. Let $m$ be the order of $g$ in $G$. Then there is a natural injective morphism of groups

$$
\zeta:\langle g\rangle \rightarrow S^{1}
$$

given by $\zeta(g)=\exp (2 \pi i / m)$.

We define the space $\mathcal{L}_{g} M$ of $g$-equivariant loops in $M$ to be the subspace of $\mathcal{L} M:=$ $\operatorname{Map}\left(S^{1} ; M\right)$ of loops $\phi$ satisfying the following equation for every $z \in S^{1}$ :

$$
\phi(z \cdot \zeta(g))=\phi(z) \cdot g \text {. }
$$


The space of cyclic equivariant loops of $M$ is defined to be simply

$$
\mathcal{L}_{G} M:=\bigsqcup_{g \in G} \mathcal{L}_{g} M \times\{g\}
$$

It is, again, naturally endowed with a $G$-action $((\phi, h) ; g) \mapsto\left(\phi g, g^{-1} h g\right)$.

The natural restriction map

$$
\Psi: \mathcal{L}_{g}(M) \longrightarrow \mathcal{P}_{g}(M)
$$

given by

$$
\gamma(t)=\phi(\exp (2 \pi i t / m))=\phi\left(\zeta(g)^{t}\right)
$$

is a diffeomorphism, and moreover it induces a $G$-equivariant diffeomorphism

$$
\Psi: \mathcal{L}_{G}(M) \longrightarrow \mathcal{P}_{G}(M) .
$$

We conclude this subsection by pointing out that as a consequence of these remarks we have the following equality

$$
\mathcal{L}_{G}(M) \times_{G} E G \simeq \mathcal{L}\left(M_{G}\right)=\mathcal{L} B \times .
$$

\subsection{Principal bundles}

In this paragraph we consider $G$-principal bundles on $S^{1}$ and their relation to the various models of the loop orbifold. We are interested in the category of $G$-principal bundles $\pi: Q \rightarrow S^{1}$ over $S^{1}$ endowed with a marked point $q_{0} \in Q$ so that $\pi\left(q_{0}\right)=$ $0 \in S^{1}$, and such that $\pi$ is a local isometry.

Whenever we have such a pair $\left(Q, q_{0}\right)$ we have a well-defined lift $\tilde{e}:[0,1] \rightarrow Q$, $\tilde{e}(0)=q_{0}$, of the exponential map $e:[0,1] \rightarrow S^{1}$ given by $t \mapsto \exp (2 \pi i t)$, making the following diagram commutative:

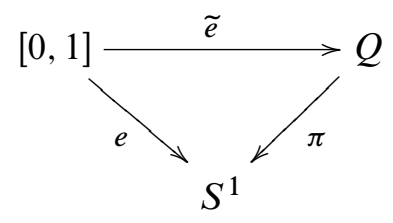

Since $\widetilde{e}(0)$ and $\widetilde{e}(1)$ belong to $\pi^{-1}(0)$ there is a $g \in G$ so that

$$
\widetilde{e}(1)=\widetilde{e}(0) \cdot g
$$

We well call this $g \in G$ the holonomy of $Q$. 
The isomorphism classes of $G$-principal bundles with a marked point are classified by their holonomy, for the set $\operatorname{Bun}_{G}\left(S^{1}\right)$ of such classes is given by

$$
\operatorname{Bun}_{G}\left(S^{1}\right)=\pi_{1} B G=G .
$$

The following proposition is very easy.

Proposition 2.7 The natural action of $G$ on $\operatorname{Bun}_{G}\left(S^{1}\right)$ under the holonomy isomorphism hol: $\operatorname{Bun}_{G}\left(S^{1}\right) \longrightarrow G$ becomes the action of $G$ on $G$ by conjugation.

This proposition can be slightly generalized as follows. Consider now the space $\operatorname{Bun}_{G}\left(S^{1}, M\right)$ of isomorphism classes of $G$-equivariant maps from a principal $G$ bundle $Q$ over the circle to $M$. This space has a natural $G$-action defined as follows. If $Q_{g}$ denotes the principal bundle with holonomy $g$ then the pair

$$
\left[\left(\beta: Q_{g} \rightarrow M\right) ; k\right] \in \operatorname{Bun}_{G}\left(S^{1}, M\right) \times G
$$

gets mapped by conjugation to

$$
\left(\beta_{k}: Q_{k^{-1} g k} \rightarrow M\right) \in \operatorname{Bun}_{G}\left(S^{1}, M\right) .
$$

Proposition 2.8 The loop orbifold $\mathrm{LX}=\left[\mathcal{P}_{G}(M) / G\right]$ is isomorphic to the orbifold $\left[\operatorname{Bun}_{G}\left(S^{1}, M\right) / G\right]$, and therefore

$$
\operatorname{Bun}_{G}\left(S^{1}, M\right) \times_{G} E G \simeq \mathcal{L}\left(M_{G}\right) .
$$

Proof It is enough to give a $G$-equivariant diffeomorphism

$$
\operatorname{Bun}_{G}\left(S^{1}, M\right) \longrightarrow \mathcal{P}_{G}(M) .
$$

This can be achieved by the following formula

$$
\left(\beta: Q_{g} \rightarrow M\right) \mapsto \gamma=\beta \circ \widetilde{e} .
$$

Since $\widetilde{e}(1)=\widetilde{e}(0) \cdot g$, then $\gamma(1)=\gamma(0) \cdot g$.

To finish this section let us define $\operatorname{Bun}_{g}\left(S^{1}, M\right)$ to be the space of isomorphism classes of $G$-equivariant maps from a principal $G$-bundle $Q_{g}$ with holonomy $g$ to $M$. Then we have that

$$
\operatorname{Bun}_{G}\left(S^{1}, M\right)=\bigsqcup_{g \in G} \operatorname{Bun}_{g}\left(S^{1}, M\right),
$$

and in fact

$$
\operatorname{Bun}_{g}\left(S^{1}, M\right) \cong \mathcal{P}_{g}(M) \text {. }
$$




\section{The orbifold string product}

\subsection{The definition of the orbifold string ring}

We will suppose at the outset of what follows that $M$ is oriented and that $G$ acts in an orientation preserving fashion. In this section we define the string product in the homology of $\mathcal{L} B X$. Let us start by defining a composition-of-paths map:

$$
\star: \mathcal{P}_{g}(M)_{\epsilon_{1}} \times_{\epsilon_{0}} \mathcal{P}_{h}(M) \longrightarrow \mathcal{P}_{g h}(M)
$$

Here the map $\epsilon_{t}: \mathcal{P}_{k}(M) \rightarrow M$ is the evaluation map at $t$ given by $\gamma \mapsto \gamma(t)$. Therefore $\mathcal{P}_{g}(M)_{\epsilon_{1}} \times \epsilon_{0} \mathcal{P}_{h}(M)$ is the space of pairs of paths $\left(\gamma_{0}, \gamma_{1}\right)$ so that $\gamma_{0}(1)=$ $\gamma_{1}(0)=: \epsilon_{\infty}\left(\gamma_{0}, \gamma_{1}\right)$, that is to say the end of $\gamma_{0}$ is the beginning of $\gamma_{1}$. The map $\star$ is given by the obvious formulas of concatenation:

$$
\left(\gamma_{0} \star \gamma_{1}\right)(t):=\left\{\begin{array}{cc}
\gamma_{0}(2 t) & \text { for } 0 \leq t \leq 1 / 2 \\
\gamma_{1}(2 t-1) & \text { for } 1 / 2<t \leq 1
\end{array}\right.
$$

Notice that the following diagram is a cartesian square:

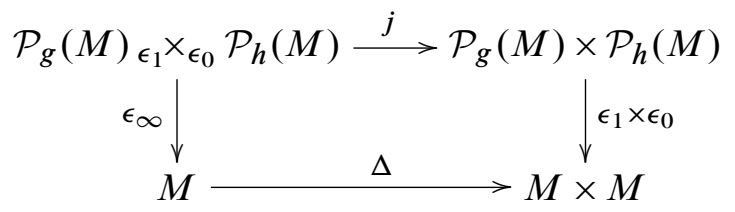

where $j$ is the natural inclusion and $\Delta$ is the diagonal map. Following Cohen and Jones [9, Section 1] we observe that such a pullback square allows one to construct a Pontrjagin-Thom map

$$
\tilde{\tau}: \mathcal{P}_{g}(M) \times \mathcal{P}_{h}(M) \longrightarrow\left(\mathcal{P}_{g}(M)_{\epsilon_{1}} \times_{\epsilon_{0}} \mathcal{P}_{h}(M)\right)^{T M}
$$

where $\left(\mathcal{P}_{g}(M) \epsilon_{1} \times_{\epsilon_{0}} \mathcal{P}_{h}(M)\right)^{T M}$ denotes the Thom space of the pullback bundle $\epsilon_{\infty}^{*}(T M)$, which is the normal bundle of the embedding $j$. Here we remind the reader that the normal bundle of the embedding $\Delta$ is $T M$.

Let us denote by $\left(\mathcal{P}_{g h}(M)\right)^{T M}$ the Thom space of the bundle $\epsilon_{1 / 2}^{*}(T M)$ where $\epsilon_{1 / 2}: \mathcal{P}_{g h}(M) \rightarrow M$. The map $\star$ induces a map of Thom spaces

$$
\widetilde{\star}:\left(\mathcal{P}_{g}(M)_{\epsilon_{1}} \times_{\epsilon_{0}} \mathcal{P}_{h}(M)\right)^{T M} \longrightarrow\left(\mathcal{P}_{g h}(M)\right)^{T M} \text {. }
$$


It is immediate to verify that the following diagram is commutative:

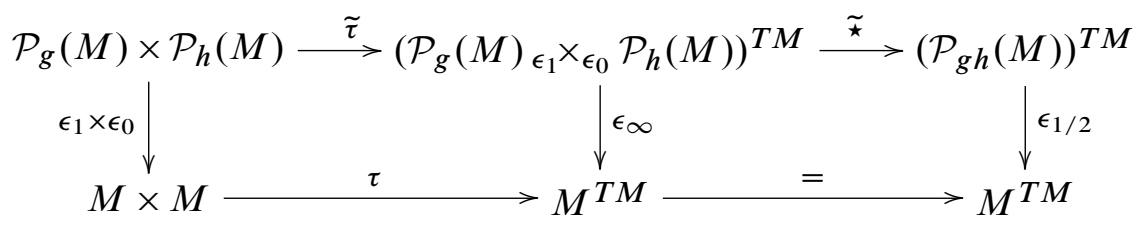

From this we can consider the composition

$$
\begin{aligned}
\circ & H_{p}\left(\mathcal{P}_{g}(M)\right) \otimes H_{q}\left(\mathcal{P}_{h}(M)\right) \stackrel{\times}{\rightarrow} H_{p+q}\left(\mathcal{P}_{g}(M) \times \mathcal{P}_{h}(M)\right) \stackrel{\tilde{\tau}_{*}}{\rightarrow} \\
H_{p+q}\left(\left(\mathcal{P}_{g}(M)_{\epsilon_{1}} \times_{\epsilon_{0}}\right.\right. & \left.\left.\mathcal{P}_{h}(M)\right)^{T M}\right) \stackrel{\tilde{\star}_{*}}{\rightarrow} H_{p+q}\left(\left(\mathcal{P}_{g h}(M)^{T M}\right) \stackrel{\tilde{u}_{*}}{\rightarrow} H_{p+q-d}\left(\mathcal{P}_{g h}(M)\right),\right.
\end{aligned}
$$

where $\tilde{u}_{*}$ is the Thom isomorphism. By considering the direct sum over all elements $g \in G$ we obtain the map

$$
\circ: H_{p}\left(\mathcal{P}_{G}(M)\right) \otimes H_{q}\left(\mathcal{P}_{G}(M)\right) \longrightarrow H_{p+q-d}\left(\mathcal{P}_{G}(M)\right) .
$$

We will call $\circ$ the $G$-string product, and $H_{*}\left(\mathcal{P}_{G}(M)\right)$ the $G$-string ring of the $G$-manifold $M$.

This ring depends on the $G$-manifold $M$ and not only on the orbifold $\mathrm{X}=[M / G]$. To obtain a bona fide orbifold invariant we proceed in the following manner.

We will define maps:

(i) The transfer map $\theta_{*}: H_{*}(\mathcal{L} B \mathrm{X})=H_{*}\left(\mathcal{P}_{G}(M) \times_{G} E G\right) \rightarrow H_{*}\left(\mathcal{P}_{G}(M)\right)$ which is the composition in homology of the transfer of the finite covering $\mathcal{P}_{G}(M) \times$ $E G \rightarrow \mathcal{P}_{G}(M) \times_{G} E G$ with the projection $\mathcal{P}_{G}(M) \times E G \rightarrow \mathcal{P}_{G}(M)$ (see Adams [2, Chapter 4]).

(ii) The projection map $\sigma_{*}: H_{*}\left(\mathcal{P}_{G}(M)\right) \rightarrow H_{*}\left(\mathcal{P}_{G}(M) \times_{G} E G\right)=H_{*}(\mathcal{L} B \mathrm{X})$ which is the composition in homology of the maps

$$
\mathcal{P}_{G}(M) \rightarrow \mathcal{P}_{G}(M) \times E G \rightarrow \mathcal{P}_{G}(M) \times_{G} E G .
$$

We then define the orbifold string product $\circ$ as follows:

$$
\begin{aligned}
\circ: H_{p}(\mathcal{L} B \mathrm{X}) \otimes H_{q}(\mathcal{L} B \mathrm{X}) \stackrel{\theta_{*} \otimes \theta_{*}}{\longrightarrow} H_{p}\left(\mathcal{P}_{G}(M)\right) \otimes H_{q}\left(\mathcal{P}_{G}(M)\right) \\
\stackrel{\circ}{\rightarrow} H_{p+q-d}\left(\mathcal{P}_{G}(M)\right) \stackrel{\sigma_{*}}{\rightarrow} H_{p+q-d}(\mathcal{L} B \mathrm{X}) .
\end{aligned}
$$

We will call the ring thus obtained

$$
\text { ○: } H_{*}(\mathcal{L} B \mathrm{X}) \otimes H_{*}(\mathcal{L} B \mathrm{X}) \longrightarrow H_{*}(\mathcal{L} B \mathrm{X})
$$


the orbifold string ring of $\mathrm{X}$. Notice that the degree of $\circ$ is $-d$ whenever the dimension of $\mathrm{X}$ is $d$.

Our next claim is that the string homology of the orbifold, it is indeed an invariant on the orbifold and not of the particular choice of groupoid that we made.

Namely, we will now prove that for a functor $F:[N / \Gamma] \rightarrow[M / G]$ between two orbifolds $[N / \Gamma]$ and $[M / G]$ that defines a Morita equivalence (for a definition of Morita equivalence see Moerdijk [19]), then their string homologies are isomorphic. Using the definition it is not hard to check that $F=(f, \rho)$ is a pair consisting of a surjective homomorphism $\rho: \Gamma \rightarrow G$ and a $\rho$-equivariant, surjective, and local diffeomorphism $f: N \rightarrow M$ such that the following diagram is a cartesian square:

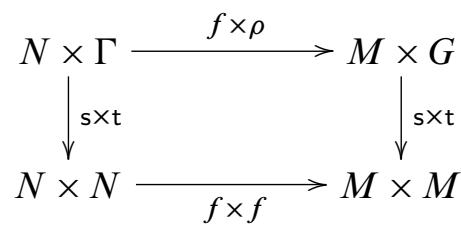

Proposition 3.1 Let $F:[N / \Gamma] \rightarrow[M / G]$ be a Morita equivalence. Then the induced homomorphism in string homologies

$$
H_{*}\left(\mathcal{L} N_{\Gamma} ; \mathbb{Q}\right) \longrightarrow H_{*}\left(\mathcal{L} M_{G} ; \mathbb{Q}\right)
$$

is an isomorphism.

Proof In [16] the first two authors that the induced map on loop orbifolds

$$
\mathrm{L} F: \mathrm{L}[N / \Gamma] \rightarrow \mathrm{L}[M / G]
$$

is a Morita equivalence. Then, their classifying spaces are homotopically equivalent and therefore the induced homomorphism in homologies is an isomorphism. We are left to prove the fact that the string homology structures are the same.

As $\mathrm{L}[N / \Gamma]=\left[\mathcal{P}_{\Gamma}(N) / \Gamma\right]$ and $\mathrm{L}[M / G]=\left[\mathcal{P}_{G}(M) / G\right]$ the induced map on the coarse moduli spaces

$$
\widetilde{F}: \mathcal{P}_{\Gamma}(N) / \Gamma \stackrel{\cong}{\rightarrow} \mathcal{P}_{G}(M) / G
$$

is a homeomorphism. Following the definition of the circle action given in Section 2.3, one can see that the map $\widetilde{F}$ is $S^{1}$-equivariant. Therefore the induced degree shifting action in homologies $H_{n} \rightarrow H_{n+1}$ is the same. Now let's see that the string product agrees. 
For $\alpha, \beta \in \Gamma$ and $a=\rho(\alpha), b=\rho(\beta)$ consider the following diagram as in (3) induced by $F$.

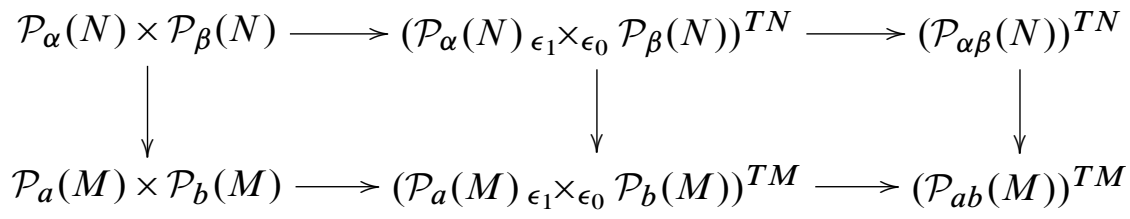

The diagram is trivially equivariant and as $T N \cong f^{*} T M$ then the diagram is commutative. Therefore once one take invariants, it produces an isomorphism of string products:

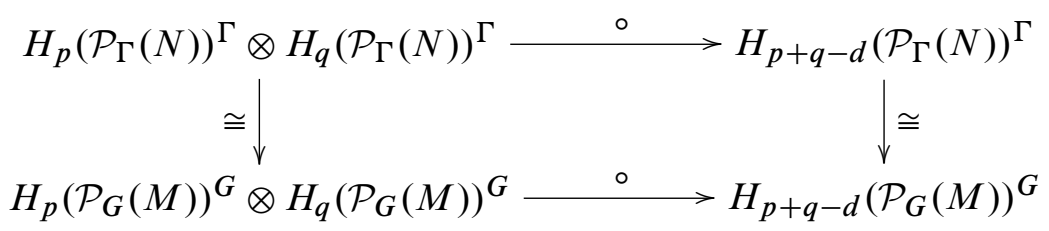

\section{Discrete torsion}

For a gerbe over the finite group $G$, namely a 2-cocycle $\alpha: G \times G \rightarrow \mathrm{U}(1)$, one can associate a 1 -cocycle over the inertia groupoid of $G$ [18]. The inertia groupoid $\wedge G$ of $G$ is the translation groupoid of the adjoint action of $G$ on itself. Its space of objects is $\wedge G_{0}=G$, its space of arrows is $\wedge G_{1}=G \times G$ and its structural maps are s $(g, h)=g$, $\mathrm{t}(g, h)=h^{-1} g h, \mathrm{~m}\left((g, h),\left(h^{-1} g h, k\right)\right)=(g, h k)$. It is Morita equivalent to the groupoid $\bigsqcup_{(g)} C(g)$ where $(g)$ runs over conjugacy classes of elements in $G$ and $C(g)$ is the centralizer of $g$.

The 1-cocycle over $\wedge G$ induced by $\alpha$ is the groupoid map:

$$
\begin{aligned}
\tau: \wedge G & \rightarrow \mathrm{U}(1) \\
(g, h) & \mapsto \frac{\alpha(g, h)}{\alpha\left(h, h^{-1} g h\right)}
\end{aligned}
$$

where $\mathrm{U}(1)$ is the groupoid with one object and morphisms the unitary complex numbers.

This 1-cocycle $\tau$ once restricted to the centralizers $C(g)$ is what is known by "discrete torsion". For every $g \in G$ it defines 1 -dimensional representation $\tau_{g}$ where the action of $h \in C(g)$ is given by the multiplication of $\tau(g, h)$. 
The loop orbifold $\mathrm{LX}:=\left[\mathcal{P}_{G}(M) / G\right]$ is Morita equivalent to the groupoid

$$
\bigsqcup_{(g)}\left[\mathcal{P}_{g}(M) / C(g)\right]
$$

As the group $G$ is finite we have that

$$
H_{*}\left(\mathcal{P}_{G}(M) \times_{G} E G ; \mathbb{C}\right) \cong H_{*}\left(\mathcal{P}_{G}(M) ; \mathbb{C}\right)^{G}
$$

where the second expression means the $G$ invariant part.

Definition 4.1 The loop orbifold homology twisted by a discrete torsion $[\alpha]$ in $H^{2}(G, \mathrm{U}(1))$ is

$$
H_{*}^{\alpha}(\mathcal{L} B \mathrm{X}):=\bigoplus_{(g)}\left[H_{*}\left(\mathcal{P}_{g}(M) ; \mathbb{C}\right) \otimes \tau_{g}\right]^{C(g)}
$$

where $C(g)$ acts by translation on $H_{*}\left(\mathcal{P}_{g}(M) ; \mathbb{C}\right)$ and $\tau_{g}$ is the $C(g)$-representation defined above. As a vector space this is isomorphic to

$$
\bigoplus_{(g)}\left[H_{*}\left(\mathcal{P}_{g}(M) ; \mathbb{C}\right)\right]^{C(g)}
$$

where for $h \in C(g)$ and $x \in H_{*}\left(\mathcal{P}_{g}(M) ; \mathbb{C}\right), h \cdot x=\tau(g, h) h_{*} x$.

\section{Operadic structure}

In [9, Section 2] Cohen and Jones showed that the BV algebra structure of the homology of free loop space of a manifold could be understood via some suitable action of the cactus operad. In this section we will argue that the homology of the orbifold loops $\mathcal{P}_{G}(M)$ can also be endowed with the action of an operad that we have named the marked $G$ chord diagram operad. The elements of this operad can be understood as $G$-principal bundles over marked chord diagrams, this last operad turns out to be homeomorphic to the cactus operad.

\subsection{Chord diagrams}

The chord diagrams we will be interested in will have only one boundary circle, will be flat (namely, the chords do not intersect each other) and will have several marked points. These marked points will be very useful when considering orbifold loops. First we define what we mean by a labeled chord diagram.

Definition 5.1 A labeled chord diagram $c$ with $n-1$ chords consists of the following information: 
- A counterclockwise oriented circle $S^{1}$ centered at the origin in $\mathbb{R}^{2}$ with perimeter 1. Namely

$$
S^{1}:=\{z \in \mathbb{C}:|z|=1 / 2 \pi\} .
$$

- We will say that $u=1 \in S^{1}$ is the initial marked point.

- Marked points $x_{i}, y_{i} \in S^{1}, i \in\{1,2, \ldots, n-1\}$ with $x_{i} \neq y_{i}$, such that they satisfy the following properties:

- The segment of line (known as the $i$-th chord) from $x_{i}$ to $y_{i}$ do not intersect the $j$-th chord in the interior of the disc (we will say in this case that the chord diagram in flat or unknotted). We will call by $f_{i}:\left\{x_{i}\right\} \rightarrow\left\{y_{i}\right\}$ the function that associates $y_{i}$ to $x_{i}$. We do this because we want to think of a chord as isomorphism between two points in the circle.

- The $n$ connected regions that the chords define inside the circle are numbered from 1 to $n$.

- The boundary of each of the $n$ connected regions that the chords define should intersect the circle $S^{1}$ in a set of measure bigger than zero.

The space of labeled chord diagrams with $n-1$ chords will be denoted by $\mathcal{E C}(n)$.

The term labeled stands for the labeling of the chords. The topology of $\mathcal{E C}(n)$ is the one inherited from $\left(S^{1}\right)^{2 n-2}$.

Remark 5.2 To have a more intuitive picture of the above construction, one could think of each chord as the place where the circle gets "pinched" to form a cactus (following the terminology of Cohen and Voronov [11, Section 2.2]); each region becomes a lobe in the cactus. The condition that the intersection of the boundary of each region with the circumference of the circle must be of measure bigger than zero ensures that each region defines a lobe of the cactus; if the boundary of a region had only isolated points of the circle, it would mean that the lobe got contracted until it disappeared. We need the number of lobes to remain fixed for each $n$ (see Figure 2). We would like to emphasize that we will not use the cactus operad in this paper, but rather an equivalent operad of chord diagrams defined in this section.

If we erase the circle and keep the chords and the $x_{i}$ 's, $y_{i}$ 's, we get a graph $\operatorname{graph}(c)$, the chords are the edges and the $x_{i}$ and $y_{i}$ 's are the vertices. The condition that the boundary of each region must contain a set of measure bigger than zero of the circle is the same as saying that $\operatorname{graph}(c)$ is a forest. A forest is a graph whose connected components are trees. A tree is a connected graph without loops. Note that the points $x_{i}$ and $y_{j}$ could be equal, but they should define a forest. All these concepts easier to understand with a picture-see Figure 3. 

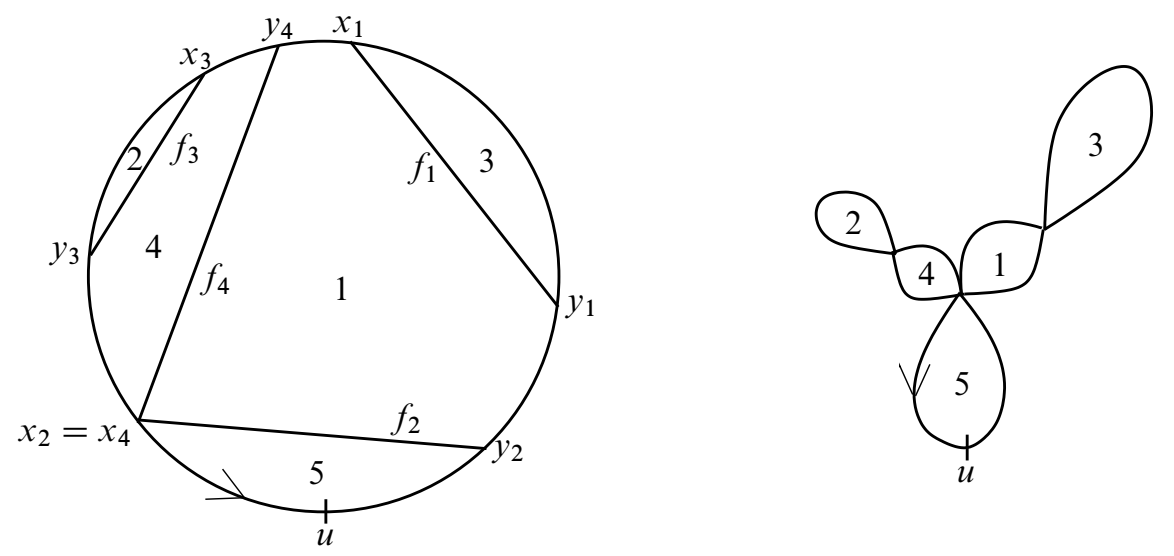

Figure 2: A labeled chord diagram and the associated cactus
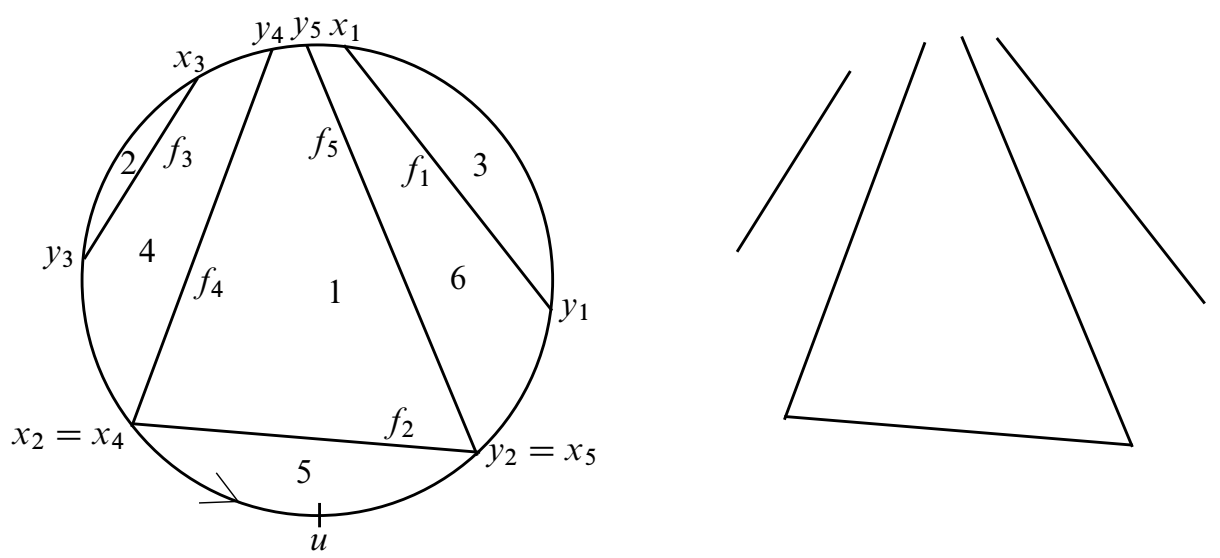

Figure 3: A labelled chord diagram and the associated forest

The group $\Gamma_{n}:=\mathfrak{S}_{n-1} \ltimes\left(\mathfrak{S}_{2}\right)^{n-1}$ acts on $\mathcal{E C}(n)$ by permuting the chords, ie each $\mathfrak{S}_{2}$ permutes the $x_{i}$ with the $y_{i}$ and the $\mathfrak{S}_{n-1}$ permutes the indices. The action is free.

Definition 5.3 The space of unlabeled chord diagrams will be denoted by $\mathcal{C}(n)$ and is defined as the quotient $\mathcal{C}(n):=\mathcal{E C}(n) / \Gamma_{n}$.

Many unlabeled chord diagrams could define the same cactus as in Figure 4. 

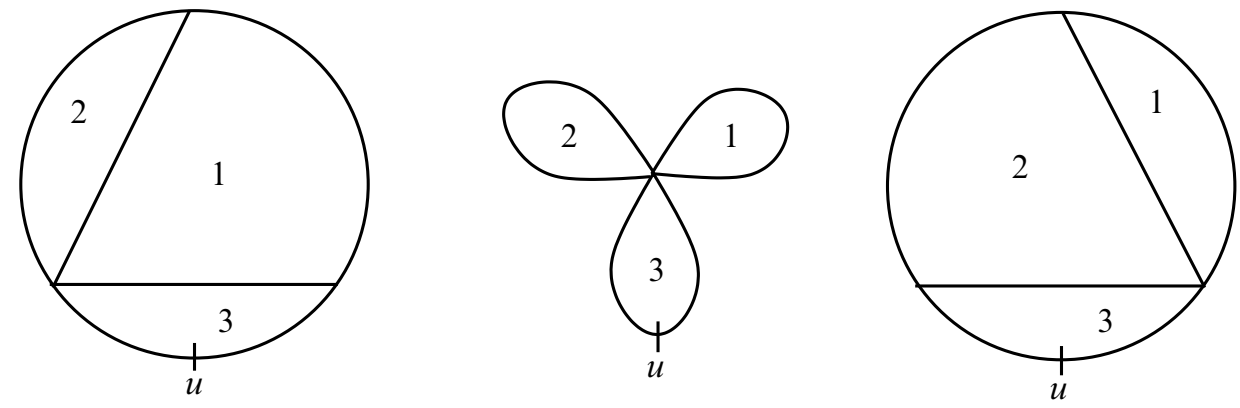

Figure 4: Two different unlabelled chord diagrams that represent the same cactus

For an unlabeled chord diagram $c \in \mathcal{C}(n)$, the vertices of the forest graph $(c)$ are divided into clusters, namely two vertices of $\operatorname{graph}(c)$ are in the same cluster if they are in the same tree.

The number of each region labels the different intervals in which the circumference of the circle is divided by the chords. Therefore one can label each such interval with the number of the region on which it lays.

Definition 5.4 Two unlabeled chord diagrams $c, c^{\prime} \in \mathcal{C}(n)$ have related forests if:

- The vertices of the forests of $\operatorname{graph}(c)$ and of $\operatorname{graph}\left(c^{\prime}\right)$ are the same, and they both define the same cluster.

- They both define the same labels for the intervals of the boundary of the circle.

We will denote this relation by $c \sim_{f} c^{\prime}$.

We have that $\sim_{f}$ is an equivalence relation. If the forest of $\operatorname{graph}(c)$ consists of $n-1$ trees with one branch, then its equivalence class has only one element.

Definition 5.5 The space of equivalence classes of unlabeled chord diagrams $\mathcal{D}(n):=$ $\mathcal{C}(n) / \sim_{f}$ will be called the space of chord diagrams.

We claim that the homotopy type of $\mathcal{D}(n)$ is the same as the set of configurations of $n$ ordered points in $\mathbb{R}^{2}$, and therefore homotopically equivalent to the space of configurations of $n$ little discs. Unfortunately the spaces $\{\mathcal{D}(n)\}_{n}$ are not endowed with an operad structure as are the little discs, for the loops defining each region do not have a marked point. 


\subsection{Marked chord diagrams}

In each of the loops determined by each of the regions of a labeled chord diagram we are going to place a marking point. This point will only be allowed to move in the loop defined by the respective region.

Definition 5.6 A marked labeled chord diagram $\mathbf{c}=\left(c,\left(z_{1}, \ldots, z_{n}\right)\right)$ is a labeled chord diagram $c$ together with marked points $z_{i} \in S^{1}, i \in\{1, \ldots, n\}$, such that $z_{i}$ lies on the closed interval of the circle with label $i$.

As we want the $z_{i}$ to move only on the $i$-th loop determined by the $i$-th region, we need to identify marked labeled chord diagrams whenever one has that $z_{i}$ equals $x_{j}$ or $y_{j}$ for some $j$. So we say that $\mathbf{c}$ and $\mathbf{c}^{\prime}$ are related, $\mathbf{c} \sim_{0} \mathbf{c}^{\prime}$, if $c=c^{\prime}$ and for all $i, z_{i} \neq z_{i}^{\prime}$ implies that both $z_{i}$ and $z_{i}^{\prime}$ are vertices of the same tree of the forest of $\operatorname{graph}(c)$. It is clear that $\sim_{0}$ is an equivalence relationship. Abusing the notation we define:

Definition 5.7 The space of marked labeled chord diagrams will be denoted by $\mathcal{M E C}(n)$ and is defined as the quotient

$$
\mathcal{M E C}(n):=\{\mathbf{c} \mid \mathbf{c} \text { is a marked labeled chord diagram }\} / \sim_{0} .
$$

See Figure 5.

Then the natural forgetful map $\mu_{n}: \mathcal{M E C}(n) \rightarrow \mathcal{E C}(n), \mathbf{c} \mapsto c$ is the quotient map by the natural action of $\left(S^{1}\right)^{n}$. Moreover, the group $\Gamma_{n}$ acts in the natural way on $\mathcal{M E C}(n)$ making the map $\mu_{n}$ into a $\Gamma_{n}$-equivariant map.

Definition 5.8 The space of marked unlabeled chord diagrams will be denoted by $\mathcal{M C}(n)$ and is defined as the quotient $\mathcal{M C}(n):=\mathcal{M E C}(n) / \Gamma_{n}$.

If furthermore we mod out by the relation $\sim_{f}$ (that is well defined in $\mathcal{M C}(n)$ because of the relationship $\sim_{0}$ ) we have the following:

Definition 5.9 The space of marked chord diagrams will be denoted by $\mathcal{M D}(n)$ and is defined as the quotient $\mathcal{M D}(n):=\mathcal{M C}(n) / \sim_{f}$.

Therefore we have the commutative diagram

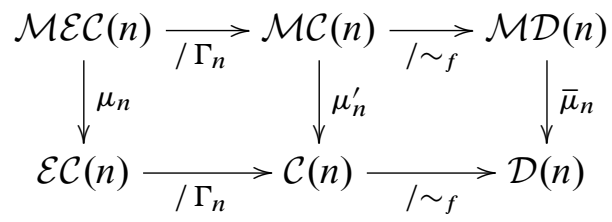




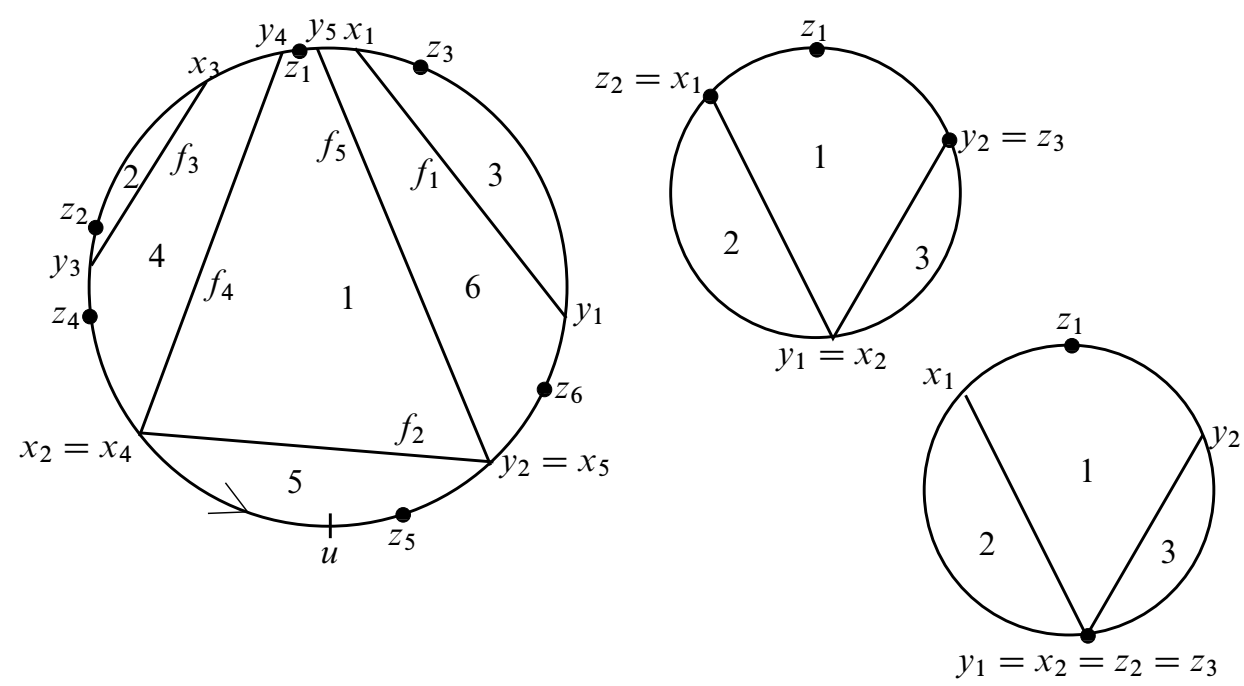

Figure 5: A marked labeled chord diagram and two configurations defining the same marked labeled chord diagram

where the forgetful maps $\mu_{n}^{\prime}: \mathcal{M C}(n) \rightarrow \mathcal{C}(n)$ and $\bar{\mu}_{n}: \mathcal{M D}(n) \rightarrow \mathcal{D}(n)$ are quotient maps by the action of $\left(S^{1}\right)^{n}$. This is true because the action of the group $\Gamma_{n}$ is free in both spaces, and because the relation $\sim_{f}$ in $\mathcal{M C}(n)$ is defined by the lift of the relation $\sim_{f}$ in $\mathcal{C}(n)$.

We will argue now that each marked unlabeled chord diagram determines uniquely a cactus (with marked points). So, let us recall the definition of the cactus operad Cac given in [9, Section 2]. A point $a$ in the space $\operatorname{Cac}(n)$ is a collection of $n$ oriented, parameterized circles $a_{1}, \ldots, a_{n}$ with radii $r_{i}$ so that $\sum_{i=1}^{n} r_{i}=1$. Each circle has a marked point $s_{i} \in a_{i}$ given by the image under the parametrization of the base point $1 \in S^{1}$. The circles can intersect each other at a finite number of points creating a "cactus type configuration", namely that the dual graph of this configuration is a tree (the dual graph of the configuration has for vertices the circles and an edge between two circles whenever they intersect, we will denote it by tree $(a)$ ). The boundary of the cactus (the union of the circles) is equipped with a basepoint $w$ together with a choice of which component the base point $w$ lies in. The edges coming into any vertex are also equipped with a cyclic ordering. The topology of the space of $\operatorname{cacti} \operatorname{Cac}(n)$ is described in $[11 ; 21]$. 
Proposition 5.10 There is a surjective map $\delta_{n}: \mathcal{M C}(n) \rightarrow \operatorname{Cac}(n)$ that factors through:

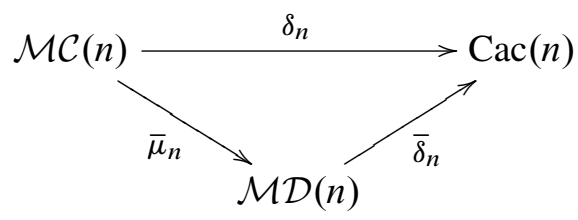

Moreover, the map $\bar{\delta}_{n}: \mathcal{M D}(n) \rightarrow \operatorname{Cac}(n)$ is a homeomorphism.

Proof A marked unlabeled chord diagram $\mathbf{c}=\left(c,\left(z_{1}, \ldots, z_{n}\right)\right)$ has $n$ connected regions in its interior, each one with a number. Once the chords of the boundary of each region are contracted, one obtains a loop $\alpha_{i}$ for each region, with certain perimeter $r_{i}$. Moreover, each loop $\alpha_{i}$ has a marked point $z_{i}$. For each $i$, take $a_{i}$ to be the parameterized circle of radius $r_{i}$ and marked point $s_{i} \in a_{i}$ (the image of $u=1 \in S^{1}$ in the parametrization), and construct the unique orientation preserving map $h_{i}: \alpha_{i} \rightarrow a_{i}$ of constant velocity and winding number 1 , such that $h_{i}\left(z_{i}\right)=s_{i}$. As the total perimeter of the loops $\alpha_{i}$ is 1 , then $\sum_{i=1}^{n} r_{i}=1$.

The intersection of the circles $a_{i}$ are obtained via the maps $h_{i}$ and the points where the chords got contracted. The cyclic order of the circles at each intersection point is given by the following algorithm. Intersection points in the cactus correspond to trees in $\operatorname{graph}(c)$. Fix a tree. Each vertex of a given tree has a small neighborhood in $S^{1}$ that has two labels. These labels in turn correspond to labels in the lobes of the cactus that intersect at the point corresponding to the tree. We will order the labels via the counterclockwise orientation of $S^{1}$ at the vertex of the tree. In this manner the lobes get a cyclic ordering.

Finally, let $j$ be the region where $u=1 \in S^{1}$ lies on. In the case that $u$ is over a vertex of the forest $\operatorname{graph}(c)$, take $j$ to be the region where $u$ would go if it followed the orientation. Then take $w:=h_{j}(u)$ lying in the circle $a_{j}$.

The cactus $a$ is uniquely determined by the marked labeled chord diagram $\mathbf{c}$, then we define $\delta_{n}(\mathbf{c}):=a$.

If two marked unlabeled chord diagrams $\mathbf{c}$ and $\mathbf{c}^{\prime}$ satisfy $\mathbf{c} \sim_{f} \mathbf{c}^{\prime}$, then they define the same cactus, ie $\delta_{n}(\mathbf{c})=\delta_{n}\left(\mathbf{c}^{\prime}\right)$. For the intersection points of the cactus are defined by each of the trees of the forests. When the trees collapse to a point, both $\mathbf{c}$ and $\mathbf{c}^{\prime}$ define the same intersection points. Then we can define $\bar{\delta}_{n}([\mathbf{c}]):=\delta_{n}(\mathbf{c})$.

To show that $\bar{\delta}_{n}$ is a homeomorphism we will construct its inverse map. Take a cactus $a$ and let $S^{1}$ be the parameterized circle of perimeter 1 with marked point $u$. Construct the unique orientation preserving map $\kappa: a \rightarrow S^{1}$ with constant velocity $1 /(2 \pi)$ that 
maps the boundary of the cactus $a$ to the circle $S^{1}$ such that $\kappa(w)=u$. This map is unique because we start in $w$ following the boundary of the cactus in the direction of the orientation. If $w$ lies in an intersection point, since we know in which lobe $w$ lies, we move through the boundary of that lobe. Once an intersection point is reached, we use the cyclic order to know where to continue. The image under $\kappa$ of the points $s_{i}$ define the numbering of the regions as well as the marked points $z_{i}$. The image under $\kappa$ of each one of the intersection points define the vertices of each of the trees of the forest. This information defines uniquely an element in $\mathcal{M D}(n)$. Therefore we have defined a continuous map $\sigma_{n}$ : $\operatorname{Cac}(n) \rightarrow \mathcal{M D}(n)$. It is straightforward to check that $\sigma_{n}$ is the inverse of $\bar{\delta}_{n}$.

Notice that if there are $n-1$ intersection points in the cactus $a$, then we have $n-1$ pairs of points in the circles $S^{1}$ and there is only one way we can fill them with trees. In this case we can find a marked unlabeled chord diagram for the cactus $a$. If there are less than $n-1$ intersection points, there is no canonical way to decide how to construct the trees of the forest of the marked unlabeled chord diagram. Nevertheless there is always a tree, take for example the linear graph defined by the cyclic order given by the cyclic orientation starting at any point in a cluster.

The spaces $\{\mathcal{M E C}(n)\}_{n \geq 0},\{\mathcal{M C}(n)\}_{n \geq 0}$ and $\{\mathcal{M D}(n)\}_{n \geq 0}$ form operads. An operad (see Voronov [21, Definition 2.3]) is a set of spaces $\{\mathcal{O}(n)\}_{n}$ with the following properties:

- A composition law:

$$
\mathcal{O}(m) \times \mathcal{O}\left(k_{1}\right) \times \cdots \times \mathcal{O}\left(k_{m}\right) \longrightarrow \mathcal{O}\left(k_{1}+\cdots+k_{m}\right)
$$

- A right action of the symmetric group $\mathfrak{S}_{n}$ on $\mathcal{O}(n)$

- A unit $e \in \mathcal{O}(1)$

such that the composition is associative and equivariant with respect to the symmetric groups, and the identity satisfies natural properties with respect to the composition.

Let's see how the operad structure is defined in $\mathcal{M E C}$. The action of $\mathfrak{S}_{n}$ is given by permutations of the subindices of the marked points $z_{i}$ (and therefore the numbering of the regions), and the composition is obtained by adding the chords of the diagrams $\mathbf{c}_{\mathbf{i}} \in \mathcal{M E C}\left(k_{i}\right)$ into the diagram of $\mathbf{c} \in \mathcal{M E C}(m)$. This is done as follows: the diagram $\mathbf{c}_{\mathbf{i}}$ will be patched into the $i$-th region (where the point $z_{i}$ lies in $\mathbf{c}$ ). $\mathbf{c}_{\mathbf{i}}$ is linearly rescaled to match the length of the $i$-th loop of $\mathbf{c}$. The patching starts by sending the marked point $u^{i}$ of $\mathbf{c}_{\mathbf{i}}$ to the point $z_{i}$ and one continues using the orientations. The points $\left\{x_{j}^{i}, y_{j}^{i}\right\}_{1 \leq j<k_{i}}$ are patched then onto $\mathbf{c}$ thus defining an 
element $\tilde{\mathbf{c}} \in \mathcal{M E C}\left(k_{1}+\cdots+k_{m}\right)$. The numbering of the chords and of the regions in $\tilde{\mathbf{c}}$ is obtained by starting with the chords and the regions in $\mathbf{c}_{\mathbf{1}}$, then the ones in $\mathbf{c}_{\mathbf{2}}$ and so forth. The marking $u$ of $\mathbf{c}$ remains the same in $\widetilde{\mathbf{c}}$. The identity $e \in \mathcal{M E C}(1)$ is the chord diagram with $z_{1}=u$.

The operad structure of $\mathcal{M E C}$ induces the operad structures on $\mathcal{M C}$ and $\mathcal{M D}$.

Theorem 5.11 The cactus operad Cac and the marked chord diagram operad $\mathcal{M D}$ are isomorphic via the maps $\bar{\delta}=\left\{\bar{\delta}_{n}\right\}_{n}$.

Proof In Proposition 5.10 we showed that the maps $\bar{\delta}_{n}: \mathcal{M E C}(n) \rightarrow \operatorname{Cac}(n)$ are homeomorphisms. The fact that they $\mathfrak{S}_{n}$ equivariant and compatible with the operad structure is straightforward.

Cohen with Godin [8] and Jones [9] showed how the homology of the Cacti operad can act on the homology of the free loop space of a manifold thus realizing the BV-algebra structure on the homology of the loops defined by Chas and Sullivan [7]. This uses the result of Voronov [21] that shows that the cactus operad is equivalent to the framed little disc operad, and the result of Getzler [14] that shows that the algebraic structure of a BV-algebra is captured by an action of the framed little disc operad.

\subsection{Marked $G$-chord diagrams}

Now we want to construct the moduli space of principal $G$ bundles over the marked chord diagrams, ie principal $G$ bundles over c for all c's. We need to lift all the markings of the marked labeled chord diagram in order to have a manifold and not an orbifold. This fact can be easily seen on the moduli space of principal $G$ bundles over a circle. If one marks a lift of 0 then the isomorphism class can be characterized by the holonomy, en element in $G$, but without the marking the only invariant we can measure is the conjugacy class of the holonomy. The first one is a manifold, the second one is an orbifold.

First, let's fix for each $g \in G$ a $G$-principal bundle $\pi: Q_{g} \rightarrow S^{1}$ (as in Section 2.5 with a marked point $\tilde{u} \in Q_{g}$, over the parameterized circle of total arclength 1 , such that $\pi$ is a local isometry, $\pi(\tilde{u})=u$ and the holonomy of $Q_{g}$ starting at $\widetilde{u}$ is $g$.

Definition 5.12 A $G$ marked labeled chord diagram $\mathbf{W}$ with holonomy $g$ over the marked labeled chord diagram $\mathbf{c} \in \mathcal{M E C}(n)$ consist of the following information:

- The principal bundle $\pi: Q_{g} \rightarrow S^{1}$

- The marked labeled chord diagram c 
- Lifts $\widetilde{z}_{1}, \ldots, \widetilde{z}_{n}$ in $Q_{g}$ for the marked points $z_{1}, \ldots, z_{n}$ in $\mathbf{c}$

- Lifts $\tilde{x}_{i}, \tilde{y}_{i} \in Q_{g}, 1 \leq i<n$ of $x_{i}$ and $y_{i}$ in $\mathbf{c}$, together with $G$-equivariant isomorphisms $\widetilde{f}_{i}: \pi^{-1}\left(x_{i}\right) \rightarrow \pi^{-1}\left(y_{i}\right), 1 \leq i<n$ such that $\tilde{f}_{i}\left(\tilde{x}_{i}\right)=\tilde{y}_{i}$.

We will denote the space of such $G$ marked labeled chord diagrams

$$
\mathbf{W}=\left(Q_{g}, \mathbf{c},\left(\tilde{x}_{i}\right)_{i},\left(\tilde{y}_{i}\right)_{i},\left(\tilde{z}_{i}\right)_{i}\right)
$$

by $G \mathcal{M E C}(n, g)$.

Considering all the possible holonomies we define $G \mathcal{M E C}(n):=\bigsqcup_{g \in G} G \mathcal{M E C}(n, g)$.

Example 5.13 In the graph, Figure 6 below, we can see a marked $\mathfrak{S}_{3}$ chord diagram over the marked labeled chord diagram of one chord. The dashed double arrows represent the isomorphism $\widetilde{f}_{1}$ and the pointed vertical lines represent the fibers of the points $x_{1}$ and $y_{1}$. We have identified the fiber of $x_{1}$ with the elements of $\mathfrak{S}_{3}$ that appear on the left of Figure 6, and the fiber of $y_{1}$ with the elements on the right.

From Figure 6 one can see that the holonomy of the outer circle (forgetting the chords) starting at $\tilde{u}$ is $(1,3,2)$, the holonomy of the region numbered 1 starting at $\widetilde{z}_{1}$ is $(2,3)$ and of the region 2 starting at $\widetilde{z}_{2}$ is also $(2,3)$. It is worth pointing out here that if one takes the $\mathfrak{S}_{3}$ principal bundle associated to either one of the regions, each one of them would have 3 connected components. Each component will give a different holonomy, namely $(1,2),(2,3)$ or $(1,3)$; all the conjugates of $(2,3)$. All these holonomies can be attained by changing the position of the points $\tilde{z}_{i}$.

Proposition 5.14 The projection maps $G \mathcal{M E C}(n, g) \rightarrow \mathcal{M E C}(n), \mathbf{W} \mapsto \mathbf{c}$, are principal $G^{3 n-2}$ bundles.

Proof There are $|G|$ choices for each of the $\tilde{x}_{i}, \tilde{y}_{i}$, and $\tilde{z}_{i}$ 's. The element

$$
\left(\left(g_{i}\right)_{i},\left(h_{i}\right)_{i},\left(k_{i}\right)_{i}\right) \in G^{n-1} \times G^{n-1} \times G^{n}
$$

sends $\mathbf{W}=\left(Q_{g}, \mathbf{c},\left(\tilde{x}_{i}\right)_{i},\left(\tilde{y}_{i}\right)_{i},\left(\tilde{z}_{i}\right)_{i}\right)$ to $\mathbf{W}^{\prime}=\left(Q_{g}, \mathbf{c},\left(\tilde{x}_{i} g_{i}\right)_{i},\left(\tilde{y}_{i} h_{i}\right)_{i},\left(\tilde{z}_{i} k_{i}\right)_{i}\right)$.

Therefore for all $g \in G$,

$$
G \mathcal{M E C}(n, g) / G^{3 n-2}=\mathcal{M E C}(n) .
$$

We want to consider now principal bundles over the marked unlabeled chord diagrams and we will use $G \mathcal{M C}(n)$ to denote this moduli space.

Consider the diagonal action

$$
\begin{aligned}
G \mathcal{M E C}(n, g) \times G^{n-1} & \rightarrow G \mathcal{M E C}(n, g) \\
\left(Q_{g}, \mathbf{c},\left(\widetilde{x}_{i}\right)_{i},\left(\tilde{y}_{i}\right)_{i},\left(\widetilde{z}_{i}\right)_{i}\right) \times\left(g_{i}\right)_{i} & \mapsto\left(Q_{g}, \mathbf{c},\left(\tilde{x}_{i} g_{i}\right)_{i},\left(\tilde{y}_{i} g_{i}\right)_{i},\left(\widetilde{z}_{i}\right)_{i}\right) .
\end{aligned}
$$


(12)

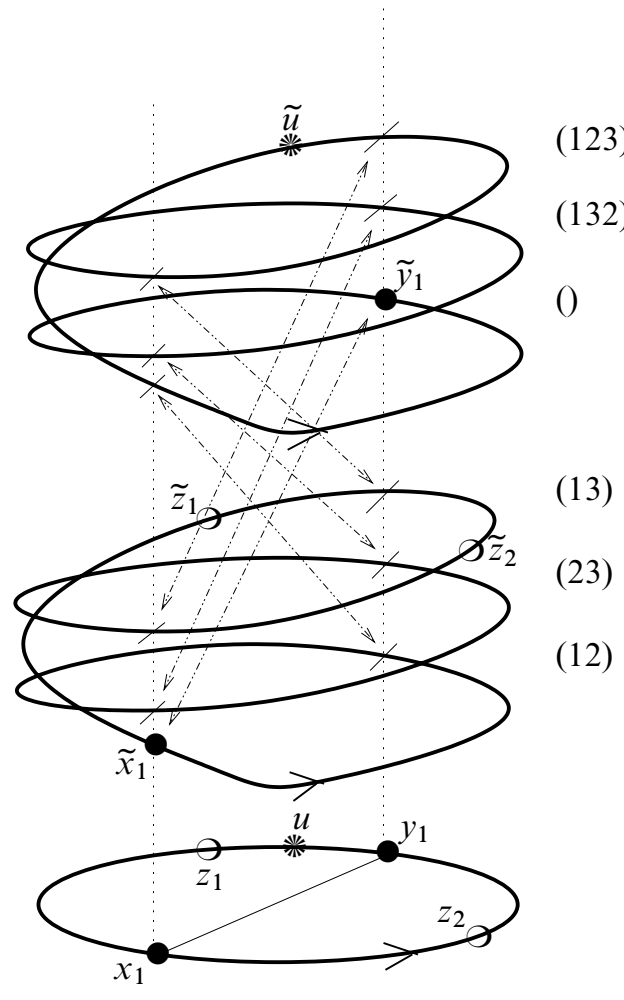

Figure 6

Definition 5.15 Let $\overline{G \mathcal{M E C}}(n, g):=G \mathcal{M E C}(n, g) / G^{n-1}$ where the $G^{n-1}$ action is the diagonal action of (5).

An equivalence class $\overline{\mathbf{W}}$ in $\overline{G \mathcal{M E C}}(n, g)$ consist of a $G$ marked labeled chord diagram where we keep track of the $G$ equivariant maps $\tilde{f}_{i}: \pi^{-1}\left(\left\{x_{i}\right\}\right) \rightarrow \pi^{-1}\left(\left\{y_{i}\right\}\right)$ lifting the $f_{i}$ 's and the $\tilde{z}_{i}$, but there are no marked points on the fibers of the $x_{i}, y_{i}$ 's.

If $G^{n-1}$ acts on $\overline{G \mathcal{M E C}}(n, g)$ by sending the maps $\left(\tilde{f}_{i}\right)_{i}$ to the maps $\left(\tilde{f}_{i}^{\prime}\right)_{i}$, where $\widetilde{f}_{i}^{\prime}(v):=\widetilde{f}_{i}(v) g_{i}$, one gets that $\overline{G \mathcal{M E C}}(n, g)$ is a $G^{2 n-1}$ principal bundle over $\mathcal{M E C}(n)$.

Over the space $\overline{G \mathcal{M E C}}(n, g)$ the group $\Gamma_{n}$ acts by permuting the $\tilde{f}_{i}$ 's lifting the action of $\Gamma_{n}$ on $\mathcal{M E C}(n)$.

Definition 5.16 The space of $G$ marked unlabeled chord diagrams with holonomy $g$, will be denoted by $\operatorname{GMC}(n, g)$ and is defined as the quotient

$$
G \mathcal{M C}(n, g):=\overline{G \mathcal{M E C}}(n, g) / \Gamma_{n} .
$$


The next lemma follows immediately from the definition.

Lemma 5.17 The forgetful map $G \mathcal{M C}(n, g) \rightarrow \mathcal{M C}(n)$ that sends the $G$ marked unlabeled chord diagram $\mathbf{W}$ to its base (a marked unlabeled chord diagram $\mathbf{c}$ ) is a finite map of order $|G|^{2 n-1}$.

Notice that there is no canonical way to get an action of the group $G^{n-1}$ on $G \mathcal{M C}(n, g)$; nevertheless $G^{n}$ acts on $G \mathcal{M C}(n, g)$ permuting the $\widetilde{z}_{i}$ 's.

An element $\mathbf{W}$ in $G \mathcal{M C}(n, g)$ consists of a principal $G$-bundle over the marked unlabeled chord diagram $\mathbf{c}$, together with $G$-equivariant maps $\pi^{-1}(a) \rightarrow \pi^{-1}(b)$, where $a, b$ are vertices of the same chord in c. The forest of graph(c) divides the vertices of the chords of $\mathbf{c}$ into clusters. The inverse image under $\pi$ in $\mathbf{W}$ of each of these clusters is further divided into $|G|$ subclusters using the functions $\left\{\widetilde{f}_{i}\right\}_{i}$. We then make the following definition:

Definition 5.18 Two $G$ marked unlabeled chord diagrams $\mathbf{W}, \mathbf{W}^{\prime} \in G \mathcal{M C}(n, g)$ are forest related $\mathbf{W} \sim_{F} \mathbf{W}^{\prime}$, if:

- Their marked unlabeled chord diagrams $\mathbf{c}, \mathbf{c}^{\prime}$ are forest related, ie $\mathbf{c} \sim_{f} \mathbf{c}^{\prime}$,

- The subclusters of the inverse image under $\pi$ of the vertices of graph(c) are the same as the connected components of the inverse image under $\pi^{\prime}$.

- $\widetilde{z}_{i}=\widetilde{z}_{i}^{\prime}$ for all $i$ (recall that the $\widetilde{z}_{i}^{\prime}$ 's as well as the $\widetilde{z}_{i}^{\prime}$ 's belong to $Q_{g}$ ).

By definition $\sim_{F}$ is an equivalence relation, so we define:

Definition 5.19 The space of $G$ marked chord diagrams with holonomy $g$, will be denoted by $G \mathcal{M D}(n, g)$ and is defined as the quotient

$$
G \mathcal{M D}(n, g):=G \mathcal{M C}(n, g) / \sim_{F} .
$$

From Lemma 5.17 and Definition 5.19 we get:

Lemma 5.20 The forgetful map $G \mathcal{M D}(n, g) \rightarrow \mathcal{M D}(n)$ that sends the $G$ marked chord diagram $\mathbf{W}$ to its base (a marked chord diagram $\mathbf{c}$ ) is finite map of order $|G|^{2 n-1}$.

Therefore we get the commutativity of the diagram

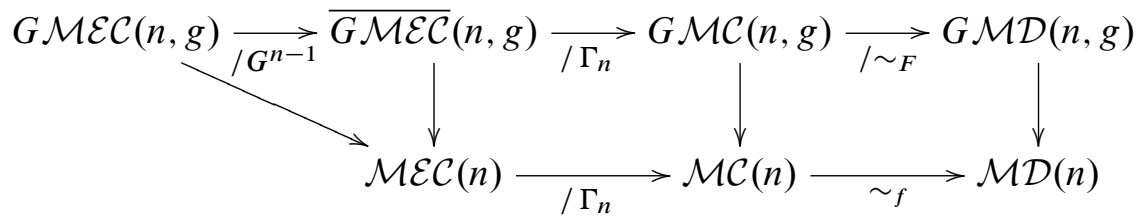


where all the vertical maps are finite or order $|G|^{2 n-1}$, the left diagonal map is a $G^{3 n-2}$ fibration, and the top horizontal maps are $G^{n}$ equivariant (by acting of the $\widetilde{z}_{i}$ 's).

We can now measure the holonomy around each of the regions that defines a marked chord diagram. Then let

$$
\text { ih: } \begin{aligned}
G \mathcal{M E C}(n, g) & \rightarrow G^{n} \\
\mathbf{W} & \mapsto\left(h_{1}, h_{2}, \ldots, h_{n}\right)
\end{aligned}
$$

be the map that assigns the holonomy around each of the $n$ regions. This is measured by starting from the points $\tilde{z}_{i}$ following the induced orientation. Whenever a point $\tilde{x}_{j}$ is reached the path is continued on $\tilde{y}_{j}$ (or viceversa) and so forth until one reaches a point on the same fiber as $\tilde{z}_{i}$. The name ih stands for incoming holonom. We will denote by

$$
\text { oh: } \begin{aligned}
G \mathcal{M E C}(n) & \rightarrow G \\
\mathbf{W} & \mapsto g
\end{aligned}
$$

the map that assigns $g$ to $\mathbf{W}$ whenever $\mathbf{W}$ belongs to $\operatorname{GMEC}(n, g)$. Recall that this is the total holonomy of $\mathbf{W}$ measured starting from $\tilde{u}$; the name oh stands for outgoing holonomy.

The incoming and outgoing holonomies are defined similarly on each of the spaces $\overline{G \mathcal{M E C}}, G \mathcal{M C}, G \mathcal{M D}$. Then we can make the next definition:

Definition 5.21 For $g, h_{1}, \ldots h_{n} \in G$ with $\mathbf{h}=\left(h_{1}, \ldots, h_{n}\right)$ let

$$
G \mathcal{M E C}(n, g, \mathbf{h}):=\left\{\mathbf{W} \in G \mathcal{M E C}(n) \mid \mathrm{ih}(\mathbf{W})=\left(h_{1}, \ldots, h_{n}\right) \text { and oh(W) }=g\right\} .
$$

Similarly for $\overline{G \mathcal{M E C}}, G \mathcal{M C}, G \mathcal{M D}$.

So, $G \mathcal{M E C}(n, g)=\bigsqcup_{\mathbf{h}} G \mathcal{M E C}(n, g, \mathbf{h})$ and similarly for $\overline{G \mathcal{M E C}}, G \mathcal{M C}, G \mathcal{M D}$. Notice that some of the sets $G \mathcal{M E C}(n, g, \mathbf{h})$ may be empty. This is because the inner holonomy $\mathbf{h}$ plus the geometric information of the bundle $\mathbf{W}$ determines uniquely the outgoing holonomy.

Fixing the incoming and outgoing holonomies we get the commutative diagram

(6)

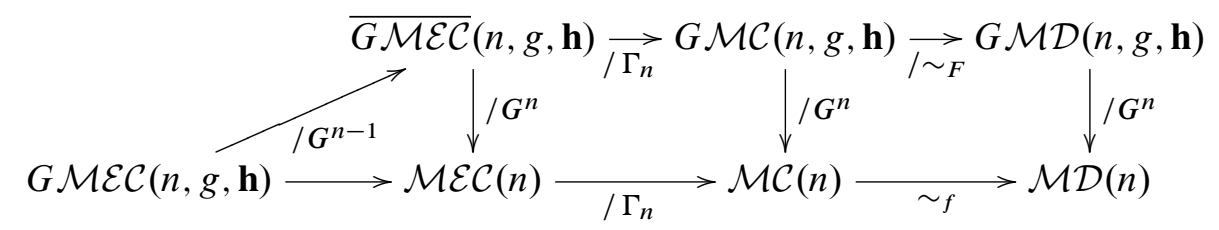

where all the vertical maps are $G^{n}$ principal bundles (the action is on the $\widetilde{z}_{i}$ 's). 
We summarize in the next lemma.

Lemma 5.22 For the spaces $G \mathcal{M D}$ we have

$$
G \mathcal{M D}(n)=\bigsqcup_{(g, \mathbf{h})} G \mathcal{M D}(n, g, \mathbf{h})
$$

and

$$
G \mathcal{M D}(n, g, \mathbf{h}) / G^{n}=\mathcal{M D}(n) .
$$

The $G$-graded operad action for $G \mathcal{M D}=\{G \mathcal{M D}(n)\}_{n}$ given by maps

$$
G \mathcal{M D}(n){ }_{\mathrm{ih}} \times\left\{\prod \mathrm{oh}\right\} \prod_{j=1}^{n} G \mathcal{M D}\left(l_{j}\right) \longrightarrow G \mathcal{M D}\left(l_{1}+l_{2}+\cdots+l_{n}\right)
$$

is defined in the natural way such that it is compatible with the one of $\{\mathcal{M D}(n)\}_{n}$, where the set $G \mathcal{M D}(n)_{\text {ih }} \times\left\{\prod_{\text {oh }} \prod_{j=1}^{n} G \mathcal{M D}\left(l_{j}\right)\right.$ is the one that makes the following a cartesian square

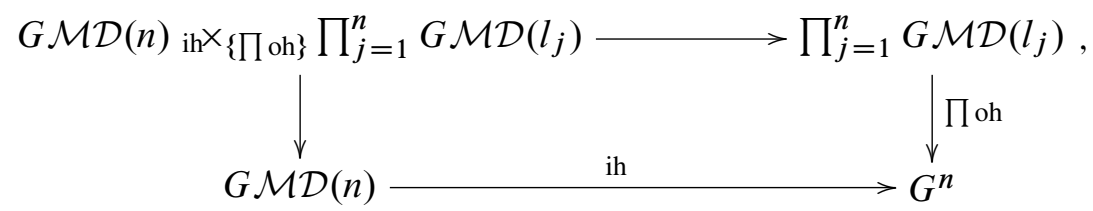

and where the identity $\tilde{e} \in G \mathcal{M D}(1)$ is the trivial $G$ principal bundle over $e \in \mathcal{M D}(1)$ with $\widetilde{z}_{i}=\tilde{u}$.

We have decided to call the structure described in Lemma 5.22 a G-graded operad. All the operads in this paper with a $G$ in their name are $G$-graded operads.

Now we are ready to construct the action of this operad in the homology of the loop orbifold.

Recall that the loop orbifold can be seen as $\left[\operatorname{Bun}_{G}\left(S^{1}, M\right) / G\right]$ where

$$
\operatorname{Bun}_{G}\left(S^{1}, M\right)=\bigsqcup_{g \in G} \operatorname{Bun}_{g}\left(S^{1}, M\right)
$$

and $\operatorname{Bun}_{g}\left(S^{1}, M\right)$ is the set of $G$-equivariant maps from $Q_{g}$ to $M$ with $Q_{g}$ the $G$-principal bundle over the circle with holonomy $g$ (measured from $q_{0}$ the lift of 0 ) and the projection being a local isometry.

Definition 5.23 For $\mathbf{W} \in G \mathcal{M E C}(n, g, \mathbf{h})$, let $L_{\mathbf{W}} M$ be

$$
L_{\mathbf{W}} M:=\left\{\gamma: Q_{g} \rightarrow M \mid \gamma \text { is } G-\text { equivariant and } \gamma\left(\tilde{x}_{i}\right)=\gamma\left(\tilde{y}_{i}\right) 1 \leq i<n\right\} .
$$


Notice that the space $L_{\mathbf{W}} M$ could also have been defined as the space of $G$ equivariant maps $\gamma: Q_{g} \rightarrow M$ such that $\gamma(\widetilde{a})=\gamma(\tilde{b})$ where $\tilde{a}, \tilde{b}$ are any two points in $Q_{g}$ lifting vertices $a, b \in \operatorname{graph}(c)$ and such that $\tilde{a}, \tilde{b}$ are in the same subcluster of $\pi^{-1}(g \operatorname{raph}(c))$ defined via the $f_{i}$ 's. Therefore we have:

Lemma 5.24 If $\mathbf{W}, \mathbf{W}^{\prime} \in G \mathcal{M E C}(n, g, \mathbf{h})$ are $G$ marked labeled chord diagram that define the same $G$ marked chord diagram $\mathbf{Z}$ (ie $\mathbf{W} \mapsto \mathbf{Z}$ and $\mathbf{W}^{\prime} \mapsto \mathbf{Z}$ under the map $G \mathcal{M E C}(n, g, \mathbf{h}) \rightarrow G \mathcal{M D}(g, n, \mathbf{h})$ of (6)), then $L_{\mathbf{W}} M$ is equal to $L_{\mathbf{W}^{\prime}} M$.

By forgetting the information about the marked points (except $\tilde{u}$ ), we get the natural map

$$
\theta_{\mathbf{W}}: L_{\mathbf{W}} M \rightarrow \operatorname{Bun}_{g}\left(S^{1}, M\right) .
$$

The space $L_{\mathbf{W}} M$ can also be viewed as the pullback of an evaluating mapping of the product $\prod_{j} \operatorname{Bun}_{h_{j}}\left(S^{1}, M\right)$ defined as follows. The marked $G$-chord diagram $\mathbf{W}$ induces $G$-principal bundles $W_{i}$ over the circles $a_{i}$ of radius $1 /(2 \pi)$ of the perimeter of the $i$-th region, by taking the $G$ principal bundle over the $i$-th region that $\mathbf{W}$ defines by contracting its chords via the maps $f_{j}$. The marked point being $\tilde{z}_{i}$.

On each of these bundles $W_{i}$ denote by $\alpha_{l}$ the points on $W_{i}$ that correspond to $\tilde{x}_{l}$ (or $\tilde{y}_{l}$ ) where $l \in I_{i}$ with $I_{i}$ the set of $j$ such that $\tilde{x}_{j}$ (or $\tilde{y}_{j}$ ) is on the $i$-th region. Let $m_{i}$ be the number of elements in $I_{i}$, and as all the chords touch two regions we have that $m_{1}+\cdots+m_{n}=2(n-1)$.

Define the evaluation map

$$
\mathrm{ev}_{\mathbf{W}}: \prod_{i=1}^{n} \operatorname{Bun}_{h_{i}}\left(S^{1}, M\right) \longrightarrow(M)^{2(n-1)}
$$

as follows. Let $s_{i}: S^{1} \rightarrow a_{i}$ be the identification of the unit circle with $a_{i}$ obtained by scaling down the unit circle so as to have the radius of $a_{i}$, and rotating it so the base point $0 \in S^{1}$ is mapped to the marked point $z_{i} \in a_{i}$. And take $\tilde{s}_{i}: Q_{h_{i}} \rightarrow W_{i}$ to be the identification of the corresponding $G$-principal bundles, compatible with $s_{i}$ and where $q_{0}$ is mapped to $\tilde{z}_{i}$. Let $\beta_{l}, l \in I_{i}$ the points on $Q_{h_{i}}$ corresponding to $\alpha_{l}, l \in I_{i}$ under the map $\tilde{s}_{i}$. Define

$$
\begin{aligned}
\operatorname{ev}_{W_{i}}: \operatorname{Bun}_{h_{i}}\left(S^{1}, M\right) & \rightarrow(M)^{m_{i}} \\
\sigma: Q_{h_{i}} \rightarrow M & \mapsto\left(\sigma\left(\beta_{j_{1}^{i}}\right), \ldots, \sigma\left(\beta_{j_{m_{i}}^{i}}\right)\right)
\end{aligned}
$$

with $j_{1}^{i}<j_{2}^{i}<\cdots<j_{m_{i}}^{i}$ all of them in $I_{i}$. 
Now define

$\mathrm{ev}_{\mathbf{W}}=\operatorname{ev}_{W_{1}} \times \cdots \times \mathrm{ev}_{W_{n}}: \prod_{i=1}^{n} \operatorname{Bun}_{h_{i}}\left(S^{1}, M\right) \rightarrow(M)^{m_{1}} \times \cdots \times(M)^{m_{n}}=(M)^{2(n-1)}$.

As each of the chords touch two regions, then each of the $j$ 's $1 \leq j<n$ belong to two of the $I_{i}$ 's. This induces a diagonal map

$$
\Delta_{\mathbf{W}}:(M)^{(n-1)} \rightarrow(M)^{m_{1}} \times \cdots \times(M)^{m_{n}}=(M)^{2(n-1)}
$$

defined by $\Delta_{\mathbf{W}}\left(p_{1}, \ldots, p_{n-1}\right)=\left(q_{j_{1}^{1}}, \ldots, q_{j_{m_{1}}^{1}}, q_{j_{1}^{2}}, \ldots, q_{j_{m_{2}}^{2}}, \ldots q_{j_{1}^{n}}, \ldots, q_{j_{m_{n}}^{n}}\right)$

with $p_{j}=q_{j_{k}^{i}}$ whenever $j=j_{k}^{i}$. As noted above, for each $j$ there are only two $j_{k}^{i}$, s that are equal to $j$.

Note that the map $\Delta_{\mathbf{W}}$ is the same as the $(n-1)$-th product of the diagonal map $M \rightarrow M \times M$ composed with a permutation of the group $\mathfrak{S}_{2(n-1)}$.

Now observe that the following is a cartesian pullback square:

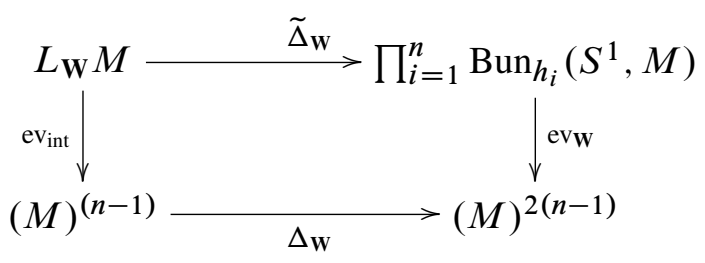

where ev int $: L_{\mathbf{W}} M \rightarrow(M)^{n-1}$ evaluates a map $\gamma: \mathbf{W} \rightarrow M$ at the $n-1$ vertices of the chords, namely evint $(\gamma)=\left(\gamma\left(\tilde{x}_{1}\right), \ldots, \gamma\left(\tilde{x}_{n-1}\right)\right)$.

The normal bundle $\eta\left(\Delta_{\mathbf{W}}\right)$ of the diagonal embedding $\Delta_{\mathbf{W}}$ is then isomorphic to $T M^{n-1} \rightarrow(M)^{n-1}$. By the tubular neighborhood theorem, we have proven the following:

Lemma 5.25 The image of the embedding

$$
\tilde{\Delta}_{\mathbf{W}}: L_{\mathbf{W}} M \rightarrow \prod_{i=1}^{n} \operatorname{Bun}_{h_{i}}\left(S^{1}, M\right)
$$

has an open neighborhood homeomorphic to the total space of the pullback $\mathrm{ev}_{\mathrm{int}}^{*} T M^{n-1}$. 
We now consider the above construction for families of diagrams by letting $\mathbf{W}$ vary in $G \mathcal{M E C}(n, g, \mathbf{h})$. Consider the set

$$
\begin{aligned}
L_{(n, g, \mathbf{h})} M=\{(\mathbf{W}, \gamma): \mathbf{W} & \left.\in G \mathcal{M E C}(n, g, \mathbf{h}) \text { and } \gamma \in L_{\mathbf{W}} M\right\}, \\
\tilde{\Delta}_{(n, g, \mathbf{h})}: L_{(n, g, \mathbf{h})} M & \rightarrow G \mathcal{M E C}(n, g, \mathbf{h}) \times \prod_{i=1}^{n} \operatorname{Bun}_{h_{i}}\left(S^{1}, M\right) \\
(\mathbf{W}, \gamma) & \mapsto\left(\mathbf{W}, \tilde{\Delta}_{\mathbf{W}}(\gamma)\right),
\end{aligned}
$$

the map

the map

$$
\begin{aligned}
\operatorname{ev}_{(n, g, \mathbf{h})}: L_{(n, g, \mathbf{h})} M & \rightarrow M^{n-1} \\
(\mathbf{W}, \gamma) & \mapsto \operatorname{ev}_{\operatorname{int}}(\gamma)=\left(\gamma\left(\widetilde{x}_{1}\right), \ldots, \gamma\left(\tilde{x}_{n-1}\right)\right),
\end{aligned}
$$

and the map

$$
\theta_{(n, g, \mathbf{h})}: L_{(n, g, \mathbf{h})} M \rightarrow \operatorname{Bun}_{g}\left(S^{1}, M\right)
$$

$$
(\mathbf{W}, \gamma) \mapsto \theta_{\mathbf{W}}(\gamma) \text {. }
$$

Defining $\xi(n, g, \mathbf{h}):=\mathrm{ev}_{(n, g, \mathbf{h})}^{*} T M^{n-1}$, the $d(n-1)$ dimensional vector bundle over $L_{(n, g, \mathbf{h})}$ whose fiber over $(\mathbf{W}, \gamma)$ is the sum of tangent spaces

$$
\left.\xi(n, g, \mathbf{h})\right|_{(\mathbf{W}, \gamma)}=\bigoplus_{i=1}^{n-1} T_{\gamma\left(\tilde{x}_{i}\right)} M
$$

together with Lemma 5.25, we have the next lemma:

Lemma 5.26 The image of the embedding

$$
\widetilde{\Delta}_{(n, g, \mathbf{h})}: L_{(n, g, \mathbf{h})} M \rightarrow G \mathcal{M E C}(n, g, \mathbf{h}) \times \prod_{i=1}^{n} \operatorname{Bun}_{h_{i}}\left(S^{1}, M\right)
$$

has an open neighborhood homeomorphic to the total space $\xi(n, g, \mathbf{h})$.

Now we need to define a similar map as in Lemma 5.26 but for the space $G \mathcal{M D}$. This we will do by stages defining it first for $\overline{G \mathcal{M E C}}$ and then on $G \mathcal{M C}$.

Recall that

$$
\overline{G \mathcal{M E C}}(n, g, \mathbf{h})=G \mathcal{M E C}(n, g, \mathbf{h}) / G^{n-1} .
$$

Take $\mathbf{W}, \mathbf{W}^{\prime} \in \operatorname{GMEC}(n, g, \mathbf{h})$ such that both map to $\mathbf{V} \in \overline{G \mathcal{M E C}}(n, g, \mathbf{h})$. By Lemma 5.24 we have that $L_{\mathbf{W}} M=L_{\mathbf{W}^{\prime}} M$, therefore we can define an action of $G^{n-1}$ on $L_{(n, g, \mathbf{h})} M$ that takes $(\mathbf{W}, \gamma) \mapsto\left(\mathbf{W}^{\prime}, \gamma\right)$ making the embedding $\widetilde{\Delta}_{(n, g, \mathbf{h})}$ $G^{n-1}$ equivariant. This action can be lifted to the vector bundle $\xi(n, g, \mathbf{h})$ by using the isomorphism

$$
\left.\xi(n, g, \mathbf{h})\right|_{(\mathbf{W}, \gamma)}=\left.\bigoplus_{i=1}^{n-1} T_{\gamma\left(\tilde{x}_{i}\right)} M \cong \xi(n, g, \mathbf{h})\right|_{\left(\mathbf{W}^{\prime}, \gamma\right)}=\bigoplus_{i=1}^{n-1} T_{\gamma\left(\tilde{x}_{i}^{\prime}\right)} M .
$$


Therefore we have that $\xi(n, g, \mathbf{h})$ becomes a $G^{n-1}$ equivariant vector bundle over $L_{(n, g, \mathbf{h})} M$.

Definition 5.27 Let $\bar{\xi}(n, g, \mathbf{h}):=\xi(n, g, \mathbf{h}) / G^{n-1}$ be the vector bundle over

$$
\bar{L}_{(n, g, \mathbf{h})} M:=L_{(n, g, \mathbf{h})} M / G^{n-1} .
$$

We have then:

Lemma 5.28 The induced map

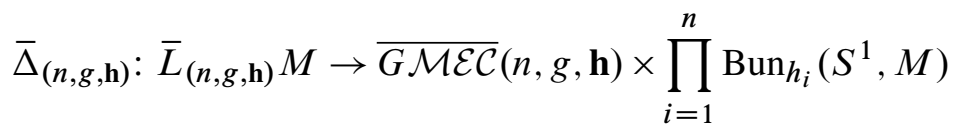

is an embedding and has an open neighborhood homeomorphic to the total space $\bar{\xi}(n, g, \mathbf{h})$.

Notice that for the vector bundle $\bar{\xi}(n, g, \mathbf{h})$, its fibers are

$$
\left.\bar{\xi}(n, g, \mathbf{h})\right|_{(\mathbf{V}, \gamma)} \cong\left(\bigoplus_{\left(g_{1}, \ldots, g_{n-1}\right) \in G^{n-1}} \bigoplus_{i=1}^{n-1} T_{\gamma\left(\tilde{x}_{i} g_{i}\right)} M\right)^{G^{n-1}}
$$

Recall that the group $\Gamma_{n}$ acts freely on $\overline{G \mathcal{M E C}}(n, g, \mathbf{h})$ and that its quotient defines $G \mathcal{M C}(n, g, \mathbf{h})$. For $\sigma \in \Gamma_{n}$, the action of $\sigma$ on $\bar{L}_{(n, g, \mathbf{h})} M$ takes $(\mathbf{V}, \gamma)$ to $(\mathbf{V} \sigma, \gamma)$ and this action can be lifted to an action on $\bar{\xi}(n, g, \mathbf{h})$ where the canonical isomorphism

$$
\begin{gathered}
\left.\left.\bar{\xi}(n, g, \mathbf{h})\right|_{(\mathbf{V}, \gamma)} \cong \bar{\xi}(n, g, \mathbf{h})\right|_{(\mathbf{V} \sigma, \gamma)} \\
\text { is }\left(\bigoplus_{\substack{\left(g_{1}, \ldots, g_{n-1}\right) \\
\in G^{n-1}}} \bigoplus_{i=1}^{n-1} T_{\gamma\left(\tilde{x}_{i} g_{i}\right)} M\right)^{G^{n-1}} \cong\left(\bigoplus_{\substack{\left.g_{1}, \ldots, g_{n-1}\right) \\
\in G^{n-1}}} \bigoplus_{i=1}^{n-1} T_{\gamma\left(\left(\tilde{x}_{i} g_{i}\right) \sigma\right)} M\right)^{G^{n-1}} .
\end{gathered}
$$

We have then that the vector bundle $\bar{\xi}(n, g, \mathbf{h})$ is $\Gamma_{n}$ equivariant.

Definition 5.29 Let $\hat{\xi}(n, g, \mathbf{h}):=\bar{\xi}(n, g, \mathbf{h}) / \Gamma_{n}$ be the vector bundle over

$$
\widehat{L}_{(n, g, \mathbf{h})} M:=\bar{L}_{(n, g, \mathbf{h})} M / \Gamma_{n} .
$$


We have then:

Lemma 5.30 The induced map

$$
\widehat{\Delta}_{(n, g, \mathbf{h})}: \widehat{L}_{(n, g, \mathbf{h})} M \rightarrow G \mathcal{M C}(n, g, \mathbf{h}) \times \prod_{i=1}^{n} \operatorname{Bun}_{h_{i}}\left(S^{1}, M\right)
$$

is an embedding and has an open neighborhood homeomorphic to the total space $\hat{\xi}(n, g, \mathbf{h})$.

Notice that for the vector bundle $\hat{\xi}(n, g, \mathbf{h})$, its fibers are

$$
\left.\hat{\xi}(n, g, \mathbf{h})\right|_{(\mathbf{Y}, \gamma)} \cong\left[\bigoplus_{\sigma \in \Gamma_{n}}\left(\bigoplus_{\left(g_{1}, \ldots, g_{n-1}\right) \in G^{n-1}} \bigoplus_{i=1}^{n-1} T_{\gamma\left(\left(\tilde{x}_{i} g_{i}\right) \sigma\right)} M\right)^{G^{n-1}}\right]^{\Gamma_{n}}
$$

where $\mathbf{V}$ maps to $\mathbf{Y}$ under the map $\overline{G \mathcal{M E C}}(n, g, \mathbf{h}) \rightarrow G \mathcal{M C}(n, g, \mathbf{h})$.

The equivalence relation $\sim_{F}$ is easily defined on $\widehat{L}_{(n, g, \mathbf{h})} M$. For $(\mathbf{Y}, \gamma),\left(\mathbf{Y}^{\prime}, \gamma^{\prime}\right) \in$ $\widehat{L}_{(n, g, \mathbf{h})} M$, we say that $(\mathbf{Y}, \gamma) \sim_{F}\left(\mathbf{Y}^{\prime}, \gamma^{\prime}\right)$ if and only if $\mathbf{Y} \sim_{F} \mathbf{Y}^{\prime}$ and $\gamma=\gamma^{\prime}$. We now want to lift this relation to $\hat{\xi}(n, g, \mathbf{h})$. Since the fibers of $\hat{\xi}(n, g, \mathbf{h})$ over $(\mathbf{Y}, \gamma)$ and $\left(\mathbf{Y}^{\prime}, \gamma\right)$ are canonically isomorphic whenever $\mathbf{Y} \sim_{F} \mathbf{Y}^{\prime}$ (this is because the images of $\gamma$ under $\mathbf{Y}$ and $\mathbf{Y}^{\prime}$ are the same) then this isomorphism defines the equivalence relation $\sim_{F}$ on $\hat{\xi}(n, g, \mathbf{h})$.

Definition 5.31 Let $\check{\xi}(n, g, \mathbf{h}):=\widehat{\xi}(n, g, \mathbf{h}) / \sim_{F}$ be the vector bundle over

$$
\check{L}_{(n, g, \mathbf{h})} M:=\widehat{L}_{(n, g, \mathbf{h})} M / \sim_{F} .
$$

And let

$$
\check{\theta}_{(n, g, \mathbf{h})}: \check{L}_{(n, g, \mathbf{h})} M \rightarrow \operatorname{Bun}_{g}\left(S^{1}, M\right)
$$

be the composition of the maps

$$
L_{(n, g, \mathbf{h})} M \longrightarrow \check{L}_{(n, g, \mathbf{h})} M \stackrel{\theta_{(n, g, \mathbf{h})}}{\longrightarrow} \operatorname{Bun}_{g}\left(S^{1}, M\right)
$$

where $\theta_{(n, g, \mathbf{h})}$ is defined in (8).

We can conclude:

Lemma 5.32 The induced map

$$
\check{\Delta}_{(n, g, \mathbf{h})}: \check{L}_{(n, g, \mathbf{h})} M \rightarrow G \mathcal{M D}(n, g, \mathbf{h}) \times \prod_{i=1}^{n} \operatorname{Bun}_{h_{i}}\left(S^{1}, M\right)
$$

is an embedding and has an open neighborhood homeomorphic to the total space $\check{\xi}(n, g, \mathbf{h})$. 
Notice that this allows us to perform a Pontrjagin-Thom collapse map

$$
\tau: G \mathcal{M D}(n, g, \mathbf{h}) \times \prod_{i=1}^{n} \operatorname{Bun}_{h_{i}}\left(S^{1}, M\right) \rightarrow\left(\check{L}_{(n, g, \mathbf{h})} M\right)^{\check{\xi}(n, \mathbf{h})}
$$

that induces a homomorphism in homology

$$
H_{p}\left(G \mathcal{M D}(n, g, \mathbf{h}) \times \prod_{i=1}^{n} \operatorname{Bun}_{h_{i}}\left(S^{1}, M\right)\right) \rightarrow H_{p-d(n-1)}\left(\check{L}_{(n, g, \mathbf{h})} M\right)
$$

that once composed with the homomorphism

$$
H_{*}\left(\check{L}_{(n, g, \mathbf{h})} M\right) \stackrel{\left(\check{\theta}_{(n, g, \mathbf{h})}\right)_{*}}{\longrightarrow} H_{*}\left(\operatorname{Bun}_{h}\left(S^{1}, M\right)\right)
$$

produces the following.

Proposition 5.33 There is a homomorphism in homology

$$
H_{p}\left(G \mathcal{M D}(n, g, \mathbf{h}) \times \prod_{i=1}^{n} \operatorname{Bun}_{h_{i}}\left(S^{1}, M\right)\right) \rightarrow H_{p-d(n-1)}\left(\operatorname{Bun}_{g}\left(S^{1}, M\right)\right)
$$

which induces an action of the homology of the $G$-graded operad $G \mathcal{M D}$.

Proof The compatibility is clear from the operadic structure of $G \mathcal{M D}$ (see (7)).

Now we are ready to show that the homology with rational coefficients of $\mathcal{L} B \mathrm{X}=\mathcal{L} M_{G}$ has a BV algebra structure, this we will prove by using the Proposition 5.33 together with the equivalences of Lemma 5.22.

Theorem 5.34 The homology with rational coefficients of the free loop space of the Borel construction $[M / G]$, ie $H_{*}\left(\mathcal{L} M_{G}, \mathbb{Q}\right)$, has a Batalin-Vilkovisky algebra structure. Moreover when the group is the identity group coincides with the ChasSullivan construction.

Proof Let's start by recalling that $\mathcal{L} M_{G}$ is homotopically equivalent to the space $\operatorname{Bun}_{G}\left(S^{1}, M\right) \times_{G} E G$, and as $G$ is finite we have that

$$
H_{*}\left(\mathcal{L} M_{G}, \mathbb{Q}\right) \cong H_{*}\left(\operatorname{Bun}_{G}\left(S^{1}, M\right) \times_{G} E G, \mathbb{Q}\right) \cong H_{*}\left(\operatorname{Bun}_{G}\left(S^{1}, M\right), \mathbb{Q}\right)^{G}
$$

where the last group means the $G$-invariant part of $H_{*}\left(\operatorname{Bun}_{G}\left(S^{1}, M\right), \mathbb{Q}\right)$. 
Using Lemma 5.22 we have the following set of maps:

$$
\begin{aligned}
& H_{*}\left(\mathcal{M C}(n) \times \prod_{i=1}^{n}\left(\mathcal{L} M_{G}\right), \mathbb{Q}\right) \cong H_{*}\left(\mathcal{M C}(n) \times \prod_{i=1}^{n}\left(\operatorname{Bun}_{G}\left(S^{1}, M\right) / G\right), \mathbb{Q}\right) \\
& \quad \longrightarrow \frac{1}{|G|^{n}} \bigoplus_{g \in G, \mathbf{h} \in G^{n}} H_{*}\left(G \mathcal{M D}(n, g, \mathbf{h}) / G^{n} \times \prod_{i=1}^{n}\left(\operatorname{Bun}_{G}\left(S^{1}, M\right) / G\right), \mathbb{Q}\right) \\
& \cong \frac{1}{|G|^{n}} \bigoplus_{g \in G, \mathbf{h} \in G^{n}} H_{*}(G \mathcal{M D}(n, g, \mathbf{h}), \mathbb{Q})^{G^{n}} \otimes \bigotimes_{i=1}^{n} H_{*}\left(\operatorname{Bun}_{G}\left(S^{1}, M\right), \mathbb{Q}\right)^{G} \\
& \longrightarrow \frac{1}{|G|^{n}} \bigoplus_{g \in G, \mathbf{h} \in G^{n}} H_{*}(G \mathcal{M D}(n, g, \mathbf{h}), \mathbb{Q}) \otimes \bigotimes_{i=1}^{n} H_{*}\left(\operatorname{Bun}_{G}\left(S^{1}, M\right), \mathbb{Q}\right)^{G} \\
& \longrightarrow H_{*}\left(\operatorname{Bun}_{G}\left(S^{1}, M\right), \mathbb{Q}\right)^{G}
\end{aligned}
$$

where the last homomorphism is the one from Proposition 5.33 whenever is defined; namely, when the inner holonomy of $\mathbf{W}$ matches the holonomy of the loops in $\prod_{i=1}^{n} \operatorname{Bun}_{G}\left(S^{1}, M\right)$ then the product is the one from Proposition 5.33, otherwise is zero.

The only thing left to prove is that the image is indeed $G$-invariant, but this should be clear from the fact that we have added over all possible $G$ marked chord diagrams on the definition of the homomorphism.

Therefore we have the set of homomorphisms

$H_{p}\left(\mathcal{M D}(n) \times \prod_{i=1}^{n}\left(\operatorname{Bun}_{G}\left(S^{1}, M\right) / G\right), \mathbb{Q}\right) \longrightarrow H_{p-d(n-1)}\left(\operatorname{Bun}_{G}\left(S^{1}, M\right) / G, \mathbb{Q}\right)$,

that are compatible with the operadic structure of $\mathcal{M D}$.

By Proposition 5.10 the chord diagram operad is isomorphic to the cactus operad, which by a theorem of Voronov [21, Theorem 2.3] is homotopy equivalent to the framed little disk operad, we have that $H_{*}\left(\mathcal{L} M_{G}, \mathbb{Q}\right)$ becomes a Batalin-Vilkovisky algebra.

When the group $G$ is the trivial group, then $\operatorname{Bun}_{G}\left(S^{1}, M\right)=\mathcal{L} M$ and $G \mathcal{M D}=\mathcal{M D}$, and we recover the homological action of the cactus operad in the homology of $\mathcal{L} M$ defined in [9].

Remark 5.35 Note that the action of $\mathcal{M D}(1)$ on

$$
\operatorname{Bun}_{G}\left(S^{1}, M\right) / G=\mathcal{P}_{G}(M) / G=\mathrm{LX} / \sim,
$$

is the one induced by the $S^{1}$ action defined on Section 2.3. 


\section{Computations}

Example 6.1 Let $M$ be a smooth manifold and consider $\mathrm{X}=[M /\{1\}]$ (in other words we consider the case when $G=\{1\})$. Then it is clear that $\mathcal{P}_{g}(M)=\mathcal{P}_{G}(M)=\mathcal{L} M$ is simple the free loop space and $H_{*}(\mathrm{LX})=H_{*}(\mathcal{L} M)$. By the work of Cohen and Jones we recover the Chas-Sullivan BV-algebra in this case.

Example 6.2 Let $G$ be a finite group and consider $\mathrm{X}=[\bullet / G]$ be the orbifold consisting of a point $M=\bullet$ being acted by $G$. Sometimes this orbifold is denoted by $\mathcal{B} G$ (not to be confused with $B G$ the classifying space of $G$ ). Clearly every loop and every path in this case is constant, namely the space $\mathcal{P}_{g}(M)=\star_{g}$ is a point, and so $\mathcal{P}_{G}(M)$ is in one-to-one correspondence with $G$. Therefore the category $\left[\mathcal{P}_{G}(M) / G\right]$ is equivalent to the category $[G / G]$ of $G$ acting on $G$ by conjugation, for we have

$$
h(\star g)=\star h g h^{-1} .
$$

For each $g \in G$ the stabilizer of this action is the centralizer

$$
C(g)=\left\{h \in G \mid h g h^{-1}=g\right\} .
$$

Now, in the category $[G / G]$ an object $g \in G$ is isomorphic to $g^{\prime} \in G$ if and only if $g$ and $g^{\prime}$ are conjugate. Therefore we have the equivalence of categories

$$
\mathrm{LX} \simeq\left[\mathcal{P}_{G}(M) / G\right] \simeq[G / G] \simeq \coprod_{(g)}\left[\star_{g} / C(g)\right]
$$

Here $(g)$ runs through the conjugacy classes of elements in $g \in G$. From this we can conclude that the equivalence

$$
\mathcal{L} B \mathrm{X}=B \mathrm{LX}
$$

becomes in this particular case ( $\mathrm{cf}[17])$

$$
\mathcal{L} B G \simeq \coprod_{(g)} B C(g) .
$$

This equation becomes at the level of homology with complex coefficients the center of the group algebra

$$
H_{*}(\mathcal{L} B G) \cong Z(\mathbb{C}[G])
$$

and in fact $H_{*}(\mathcal{L} B G)$ is simply the Frobenius algebra of Dijkgraaf and Witten [12].

The reader may be interested in comparing this result with that of Abbaspour, Cohen and Gruher[1]. 
Let $X$ be a topological space endowed with the action of a connected Lie group $\Gamma$. Take $G \subset \Gamma$ finite and consider the quotient $X / G$ and the map $\pi: X \rightarrow X / G$ the projection.

Lemma 6.3 The projection map induces an isomorphism

$$
\pi_{*}: H_{*}(X ; \mathbb{Q}) \stackrel{\cong}{\rightarrow} H_{*}(X / G ; \mathbb{Q}) .
$$

Proof Take $g \in G$ and its induced action $g: X \rightarrow X$. We claim that $g_{*}: H_{*}(X) \stackrel{\equiv}{\rightarrow}$ $H_{*}(X)$ is the identity. Join the identity of $\Gamma$ with $g$ with a path $\alpha_{t} \in \Gamma$ (ie $\alpha_{0}=\operatorname{id}_{\Gamma}$ and $\alpha_{1}=g$ ), hence $\alpha_{t}$ is a homotopy between the identity and $g$, therefore $g_{*}=$ id.

Taking the averaging operator

$$
\begin{aligned}
H_{*}(X ; \mathbb{Q}) & \stackrel{\alpha}{\rightarrow} H_{*}(X ; \mathbb{Q})^{G} \\
x & \mapsto\left(\frac{1}{|G|} \sum_{g \in G} g_{*} x\right)(=x)
\end{aligned}
$$

and using that $H_{*}(X ; \mathbb{Q})^{G} \stackrel{\iota}{\cong} H_{*}(X / G ; \mathbb{Q})$ the isomorphism follows, for it is not hard to check that $\pi_{*}=\iota \alpha$.

With the same hypothesis as before consider now the orbifold loops, namely $\mathcal{P}_{g} X=$ $\{f:[0,1] \rightarrow X \mid f(0) g=f(1)\}$.

Lemma 6.4 There is a $C(g)$-equivariant homotopy equivalence between $\mathcal{L} X$ and $\mathcal{P}_{g} X$.

Proof Let $\alpha_{t}:[0,1] \rightarrow G$ be the map defined in Lemma 6.3. Consider the maps

$$
\rho: \mathcal{P}_{g} X \rightarrow \mathcal{L} X \quad \text { and } \quad \tau: \mathcal{L} X \rightarrow \mathcal{P}_{g} X
$$

where

$$
\rho(f)(s):=\left\{\begin{array}{cl}
f(2 s) & \text { if } 0 \leq s \leq \frac{1}{2} \\
f(1) \alpha_{2 s-1}^{-1} & \text { if } \frac{1}{2} \leq s \leq 1
\end{array}\right.
$$

and

$$
\tau(\sigma)(s):=\left\{\begin{array}{cl}
\sigma(2 s) & \text { if } 0 \leq s \leq \frac{1}{2} \\
\sigma(1) \alpha_{2 s-1} & \text { if } \frac{1}{2} \leq s \leq 1 .
\end{array}\right.
$$

The composition $\rho \circ \tau: \mathcal{L} X \rightarrow \mathcal{L} X$ is clearly homotopic to the identity. The same holds for $\tau \circ \rho$. The maps $\rho$ and $\tau$ are trivially $C(g)$-equivariant.

Corollary 6.5 The group structure of the loop homology of $[X / G]$ can be seen as

$$
H_{*}(\mathrm{~L}[X / G] ; \mathbb{Q}) \cong \bigoplus_{(g)} H_{*}(\mathcal{L} X ; \mathbb{Q}) .
$$


Proof It follows from the Lemma 6.3 and Lemma 6.4 and the fact that

$$
H_{*}(\mathrm{~L}[X / G] ; \mathbb{Q}) \cong \bigoplus_{(g)} H_{*}\left(\mathcal{P}_{g} X / C(g) ; \mathbb{Q}\right)
$$

Notation Let $\mathrm{X}$ be an orbifold of dimension $d$. Denote the loop homology of $\mathrm{X}$ by

$$
\mathbb{H}_{*}(\mathrm{X}):=H_{*+d}(\mathrm{LX}) .
$$

In this way the orbifold string product $\mathbb{H}_{*}(\mathrm{X})$ is graded associative.

Example 6.6 The loop homology of the lens spaces $L_{(n, p)}=S^{n} / \mathbb{Z}_{p}(n$ odd, $p>0)$ is

$$
\mathbb{H}_{*}\left(L_{(n, p)}\right)=H_{*}\left(\mathcal{L} L_{(n, p)}\right)=\Lambda[a] \otimes \mathbb{Q}[u, v] /\left(v^{p}=1\right)
$$

with $a \in \mathbb{H}_{-n}\left(L_{(n, p)}\right), v \in \mathbb{H}_{0}\left(L_{(n, p)}\right)$ and $u \in \mathbb{H}_{n-1}\left(L_{(n, p)}\right)$.

Proof As the action of $\mathbb{Z}_{p}$ on $S^{n}$ comes from the action of $S^{1}$ on $S^{n}$ via the Hopf fibration, we can use corollary . Let $g$ be a generator of $\mathbb{Z}_{p}$, then

$$
\mathbb{H}_{*}\left(L_{(n, p)}\right) \cong \mathbb{H}_{*}\left(\left[S^{n} / \mathbb{Z}_{p}\right]\right) \cong \bigoplus_{j=0}^{p-1} H_{*}\left(\mathcal{P}_{g^{j}} S^{n}\right)^{\mathbb{Z}_{p}},
$$

as graded vector spaces.

As $H_{*}\left(\mathcal{P}_{g^{j}} S^{n}\right)^{\mathbb{Z}_{p}} \cong H_{*}\left(\mathcal{P}_{g^{j}} S^{n}\right)$ the string product $\circ$ could be calculated from the following commutative diagram:

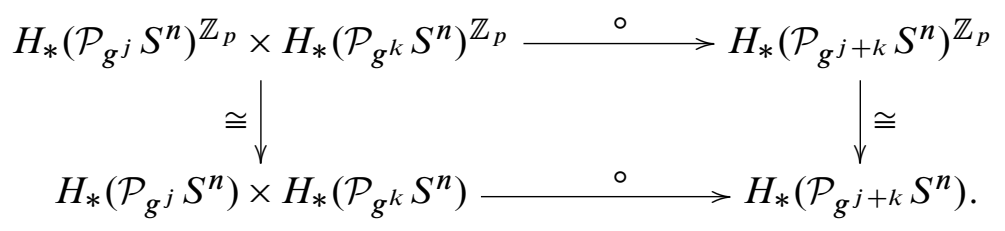

The map $\tau^{j}: \mathcal{L} S^{n} \rightarrow \mathcal{P}_{g^{j}} S^{n}$ defined in (9) gives an isomorphism in homology, so we can define the generators of the homology of $\mathcal{P}_{g^{j}} S^{n}$ via the map $\tau^{j}$ and the loop homology of the sphere, namely $\mathbb{H}_{*}\left(S^{n}\right)=H_{*}\left(\mathcal{L} S^{n}\right) \cong \Lambda[a] \otimes \mathbb{Q}[u]$ (see Cohen, Jones and Yan [10]). Denote then by $\sigma_{k}^{j}$ the generator of the group $H_{k+n}\left(\mathcal{P}_{g^{j}} S^{n}\right)$ and using that $\left(\tau^{j}\right)_{*}$ is an isomorphism one gets that

$$
\sigma_{(n-1) l-n}^{j}=\tau_{*}^{j}\left(a u^{l}\right)
$$

$\sigma_{(n-1) l}^{j}=\tau_{*}^{j}\left(u^{l}\right)$ and $\sigma_{m}^{j}=0$ for all other values of $m$. 
We claim now that

$$
\sigma_{l}^{j} \circ \sigma_{m}^{k}=\sigma_{l+m}^{j+k}
$$

The identity follows from the fact that

$$
\sigma_{l}^{j} \circ \sigma_{m}^{k}=\tau_{*}^{j}\left(\sigma_{l}^{0}\right) \circ \tau_{*}^{k}\left(\sigma_{m}^{0}\right)=\tau_{*}^{j+k}\left(\sigma_{l}^{0} \circ \sigma_{m}^{0}\right)=\tau_{*}^{j+k}\left(\sigma_{l+m}^{0}\right)=\sigma_{l+m}^{j+k}
$$

where the second identity follows from the definition of the maps $\tau$ and the third identity follows from the algebraic structure of $\Lambda[a] \otimes \mathbb{Q}[u]$.

From this we can deduce that the map $\tau_{j *}: H_{*}\left(\mathcal{L} S^{n}\right) \rightarrow H_{*}\left(\mathcal{P}_{g^{j}} S^{n}\right)$ maps $\sigma_{k}^{0} \mapsto$ $\sigma_{k}^{0} \circ \sigma_{0}^{j}$ where $\sigma_{0}^{j}$ is the $n$-simplex of paths that to every $x$ in $S^{n}$ assigns the path that goes from $x$ to $x g^{j}$ through the $S^{1}$ action.

We are only left to prove that when $j+k=p$ the formula $\sigma_{l}^{j} \circ \sigma_{m}^{k}=\sigma_{l+m}^{0}$ holds. So, let $\beta: S^{n} \rightarrow \mathcal{L} S^{n}$ be the map that to a point $x$ in the sphere associates the free loop defined that starts and ends in $x$ and travels in the direction of the $S^{1}$ action. Now define the map $\phi: \mathcal{L} S^{n} \rightarrow \mathcal{L} S^{n}$ that takes a loop $\gamma$ to $\gamma \circ \beta$. The map $\phi$ is homotopic to the identity because the cycle $\beta$ is homotopic to the cycle of constant loops over the sphere (one way to prove this uses the fact that the odd dimensional spheres have two orthogonal never vanishing vector fields). Therefore we have that $\tau^{p}: \mathcal{L} S^{n} \rightarrow \mathcal{P}_{g} S^{n}=\mathcal{L} S^{n}$ is homotopic to the identity.

We can conclude then that the elements $a=\sigma_{-n}^{0}, v=\sigma_{0}^{1}$ and $u=\sigma_{n-1}^{0}$ generate the loop homology of $\left.L_{(n, p)}\right)$, and the only extra condition is that $v^{p}=1$. Therefore

$$
\mathbb{H}_{*}\left(L_{(n, p)}\right)=\Lambda[a] \otimes \mathbb{Q}[u, v] /\left(v^{p}=1\right) .
$$

Example 6.7 Take the orbifold defined by the action of $\mathbb{Z}_{p}$ onto $S^{2}$ given by rotation of $2 \pi / p$ radians with respect to the $z$-axis. Then the loop homology of $\left[S^{2} / \mathbb{Z}_{p}\right]$ is

$$
\mathbb{H}_{*}\left(\left[S^{2} / \mathbb{Z}_{p}\right]\right)=\Lambda[b] \otimes \mathbb{Q}[a, v, y] /\left(a^{2}, a b, a v, y^{p}-1\right) .
$$

Proof The action of $\mathbb{Z}_{p}$ comes from the $S^{1}$ action on $S^{2}$ given by rotation about the $z$-axis. therefore the calculation of the loop homology product follows the same argument as in the Example 6.6. To make the notation simpler we will work with $p=2$ $\left(\mathbb{Z}_{2}=\{1, g\}\right)$; the other cases are similar.

From [10] we know that the loop homology of $S^{2}$ is given by

$$
\mathbb{H}_{*}\left(S^{2}\right)=\Lambda[b] \otimes \mathbb{Z}[a, v] /\left(a^{2}, a b, 2 a v\right)
$$

with $|b|=1,|a|=-2,|v|=2$. Since $\tau: \mathcal{L} S^{2} \rightarrow \mathcal{P}_{g} S^{2}$ is a homotopy equivalence, we will follow the argument of Example 6.6. The only different argument is on the behavior of the map $\phi:=\tau^{2}: \mathcal{L} S^{2} \rightarrow \mathcal{L} S^{2}$. In homology, $\phi_{*}$ maps $\alpha \in H_{k}\left(\mathcal{L} S^{2}\right)$ to 
$\alpha \circ \beta \in H_{k}\left(\mathcal{L} S^{2}\right)$ where $\beta \in H_{2}\left(\mathcal{L} S^{2}\right)=\mathbb{H}_{0}\left(S^{2}\right)$ is the class of the map $S^{2} \rightarrow \mathcal{L} S^{2}$ that assigns to every point $x$ the loop that starts at $x$ and rotates around the $z$ axis, and $\circ$ is the homology string product.

We claim that $\beta=1+a v$ in the notation of (10), (the proof of this fact will be postponed to Lemma 6.8). As $a v$ is a torsion class, ie $2 a v=0$, then in rational homology $\phi_{*}$ is the identity map. As in Example 6.6, we can add a new variable $y$ that behaves like a root of unity, and we conclude that

$$
\mathbb{H}_{*}\left(\left[S^{2} / \mathbb{Z}_{2}\right]\right)=\Lambda[b] \otimes \mathbb{Q}[a, v, y] /\left(a^{2}, a b, a v, y^{2}-1\right) .
$$

Lemma 6.8 The homology class $\beta \in H_{2}\left(\mathcal{L} S^{2}\right)=\mathbb{H}_{0}\left(S^{2}\right)$ of the map $S^{2} \rightarrow \mathcal{L} S^{2}$ that to a point $x$ assigns the loop that starts at $x$ and winds around the sphere once by the $S^{1}$ action, and the homology class $1+a v \in H_{2}\left(\mathcal{L} S^{2}\right)=\mathbb{H}_{0}\left(S^{2}\right)$ as in (10), are equal.

Proof When we contract all the loops of $\beta$ through the north pole we end up with the homology class $\left[S^{2}\right]+\xi$, where $\left[S^{2}\right]$ is the fundamental class of the sphere (constant loops) and therefore the unit in $1=\left[S^{2}\right] \in \mathbb{H}_{0}\left(S^{2}\right)$, and $\xi$ is defined in what follows. For $\theta \in S^{1}$ and $P_{S}$ the south pole, consider the map $f: S^{1} \times S^{1} \rightarrow \mathcal{L} S^{2}$ such that the function $f_{\theta}=f(\cdot, \theta): S^{1} \rightarrow \mathcal{L} S^{2}$ is the loop of based loops that starts at the constant loop in $P_{S}$ and goes around the sphere (as a rubber band) at the angle $\theta$. The class $f_{\theta *}\left(\left[S^{1}\right]\right)$ is the generator of $H_{1}\left(\mathcal{L} S^{2}\right)$, and the class $f_{*}\left(\left[S^{1} \times S^{1}\right]\right)$ is $\xi$. We claim that $\xi=a v$.

We know that the homology spectral sequence of the Serre fibration $\Omega S^{2} \rightarrow \mathcal{L} S^{2} \rightarrow S^{2}$ has for $E_{2}$-term

$$
E_{2}^{p, q}=H_{p}\left(S^{2}\right) \otimes H_{q}\left(\Omega S^{2}\right)
$$

with nontrivial differential $d^{2}\left(u \otimes x^{2 k+1}\right)=2 \iota \otimes x^{2 k+2}$ where $x \in H_{1}\left(\Omega S^{2}\right), \iota \in$ $H_{0}\left(S^{2}\right), 1_{\Omega} \in H_{0}\left(\Omega S^{2}\right)$ and $u \in H_{2}\left(S^{2}\right)$ are generators respectively. Also we know from [10] that $a v=\iota \otimes x^{2}$.

Denote by $\dot{T} S^{2} \stackrel{\pi}{\longrightarrow} S^{2}$ the sphere bundle of the tangent bundle $T S^{2} \rightarrow S^{2}$. The map $\pi$ is an $S^{1}$-fibration and a point in $\dot{T} S^{2}$ consists of a pair $(z, v)$ where $z \in S^{2}$ and $v$ is a unit vector tangent to $S^{2}$ at $z$. For each point $(z, v)$ we can define a map $h_{(z, v)}: S^{1} \rightarrow \mathcal{L} S^{2}$ in the same way that the function $f_{\theta}$ was defined two paragraphs above; namely, $h_{(z, v)}$ is the loop of loops that starts with the constant loop at $z$ and sweeps the sphere as a rubber band, following the direction of the oriented maximum circle tangent to the vector $v$. We can assemble all the functions $h_{(z, v)}$ by letting $(z, v)$ vary and we can obtain a function

$$
\psi: S^{1} \times \dot{T} S^{2} \rightarrow \mathcal{L} S^{2}
$$


such that $\psi(\phi,(z, v))=h_{(z, v)}(\phi)$.

The map $\psi$ defines a map of Serre fibrations

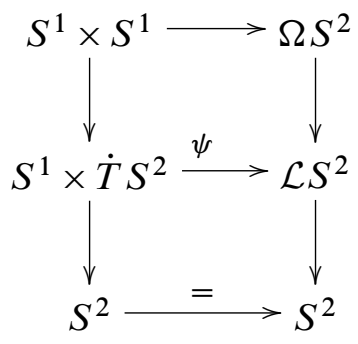

that induces a map in spectral sequences. If $\epsilon \in H_{0}\left(S^{1}\right) \otimes H_{0}\left(S^{1}\right), a \in H_{1}\left(S^{1}\right) \otimes$ $H_{0}\left(S^{1}\right), b \in H_{0}\left(S^{1}\right) \otimes H_{1}\left(S^{1}\right), c \in H_{1}\left(S^{1}\right) \otimes H_{1}\left(S^{1}\right)$, are the generators in homology, at the second term of the map of spectral sequences

$$
\psi_{*}: H_{p}\left(S^{2}\right) \otimes H_{q}\left(S^{1} \times S^{1}\right) \rightarrow H_{p}\left(S^{2}\right) \otimes H_{q}\left(\Omega S^{2}\right)
$$

induces the following identities:

- $\psi_{*}(\epsilon)=1_{\Omega}$

- $\psi_{*}(b)=0$

- $\psi_{*}(a)=x$ because the functions $f_{\theta}$ determine the generator $x$ of $H_{1}\left(\Omega S^{2}\right)$.

We also know that $d^{2}(u \otimes a)=2(\iota \otimes c)$ because $\dot{T} S^{2}=S O(3)$ and its fundamental group is $\mathbb{Z}_{2}$.

Therefore we have the following set of identities:

$$
\begin{aligned}
2\left(\iota \otimes x^{2}\right) & =d^{2}(u \otimes x) \\
& =d^{2}\left(\psi_{*}(u \otimes a)\right) \\
& =\psi_{*}\left(d^{2}(u \otimes a)\right) \\
& =\psi_{*} 2(\iota \otimes c)
\end{aligned}
$$

and this implies that $\psi_{*}(\iota \otimes c)=\iota \otimes x^{2}$. Since $\iota \otimes c$ represents the class $\left[S^{1} \times S^{1}\right]$ we can conclude that $f_{*}\left(\left[S^{1} \times S^{1}\right]\right)=\psi_{*}(\iota \otimes c)=\iota \otimes x^{2}=a v$.

\section{References}

[1] H Abbaspour, R L Cohen, K Gruher, String topology of Poincaré duality groups, from: "Proceedings of the conference on groups, homotopy and configuration spaces", (N Iwase et al, editor), Geom. Topol. Monogr. 13, Geom. Topol. Publ., Coventry (2008) $1-10$ 
[2] J F Adams, Infinite loop spaces, Annals of Math. Studies 90, Princeton Univ. Press (1978) MR505692

[3] A Adem, J Leida, Y Ruan, Orbifolds and stringy topology, Cambridge Tracts in Math. 171, Cambridge Univ. Press (2007) MR2359514

[4] A Adem, Y Ruan, Twisted orbifold K-theory, Comm. Math. Phys. 237 (2003) 533556 MR1993337

[5] I A Batalin, G A Vilkovisky, Existence theorem for gauge algebra, J. Math. Phys. 26 (1985) 172-184 MR776145

[6] M R Bridson, A Haefliger, Metric spaces of non-positive curvature, Grund. der Math. Wissenschaften [Fund. Princ. of Math. Sciences] 319, Springer, Berlin (1999) MR1744486

[7] M Chas, D Sullivan, String topology arXiv:math.GT/9911159

[8] R L Cohen, V Godin, A polarized view of string topology, from: "Topology, geometry and quantum field theory", London Math. Soc. Lecture Note Ser. 308, Cambridge Univ. Press (2004) 127-154 MR2079373

[9] R L Cohen, JD S Jones, A homotopy theoretic realization of string topology, Math. Ann. 324 (2002) 773-798 MR1942249

[10] R L Cohen, J D S Jones, J Yan, The loop homology algebra of spheres and projective spaces, from: "Categorical decomposition techniques in algebraic topology (Isle of Skye, 2001)”, Progr. Math. 215, Birkhäuser, Basel (2004) 77-92 MR2039760

[11] R L Cohen, A A Voronov, Notes on string topology, from: "String topology and cyclic homology", Adv. Courses Math. CRM Barcelona, Birkhäuser, Basel (2006) 1-95 MR2240287

[12] R Dijkgraaf, E Witten, Topological gauge theories and group cohomology, Comm. Math. Phys. 129 (1990) 393-429 MR1048699

[13] A Dold, Partitions of unity in the theory of fibrations, Ann. of Math. (2) 78 (1963) 223-255 MR0155330

[14] E Getzler, Batalin-Vilkovisky algebras and two-dimensional topological field theories, Comm. Math. Phys. 159 (1994) 265-285 MR1256989

[15] J D S Jones, Cyclic homology and equivariant homology, Invent. Math. 87 (1987) 403-423 MR870737

[16] E Lupercio, B Uribe, Loop groupoids, gerbes, and twisted sectors on orbifolds, from: "Orbifolds in mathematics and physics (Madison, WI, 2001)", Contemp. Math. 310, Amer. Math. Soc. (2002) 163-184 MR1950946

[17] E Lupercio, B Uribe, Inertia orbifolds, configuration spaces and the ghost loop space, Q. J. Math. 55 (2004) 185-201 MR2068317 
[18] E Lupercio, B Uribe, Holonomy for gerbes over orbifolds, J. Geom. Phys. 56 (2006) 1534-1560 MR2240409

[19] I Moerdijk, Orbifolds as groupoids: an introduction, from: "Orbifolds in mathematics and physics (Madison, WI, 2001)", Contemp. Math. 310, Amer. Math. Soc. (2002) 205-222 MR1950948

[20] G Segal, Classifying spaces and spectral sequences, Inst. Hautes Études Sci. Publ. Math. (1968) 105-112 MR0232393

[21] A A Voronov, Notes on universal algebra, from: "Graphs and patterns in mathematics and theoretical physics", Proc. Sympos. Pure Math. 73, Amer. Math. Soc. (2005) 81-103 MR2131012

EL, MAX: Departamento de Matemáticas, CINVESTAV

Apartado Postal 14-740, 07000 México, DF México

BU: Departamento de Matemáticas, Universidad de los Andes

Carrera 1 N. 18A - 10, Bogotá, Colombia

lupercio@math.cinvestav.mx, buribe@uniandes.edu.co,

xico@math. cinvestav.mx

Proposed: Ralph Cohen

Received: 22 January 2007

Seconded: Jim Bryan, Ron Stern

Revised: 8 July 2007

Geometry $\&$ Topology, Volume 12 (2008) 